\title{
Polarizability Corrections in Stimulated Raman Propagation
}

\author{
B. W. Shore \\ M. A. Johnson \\ S. Lowder
}

July 30, 1991

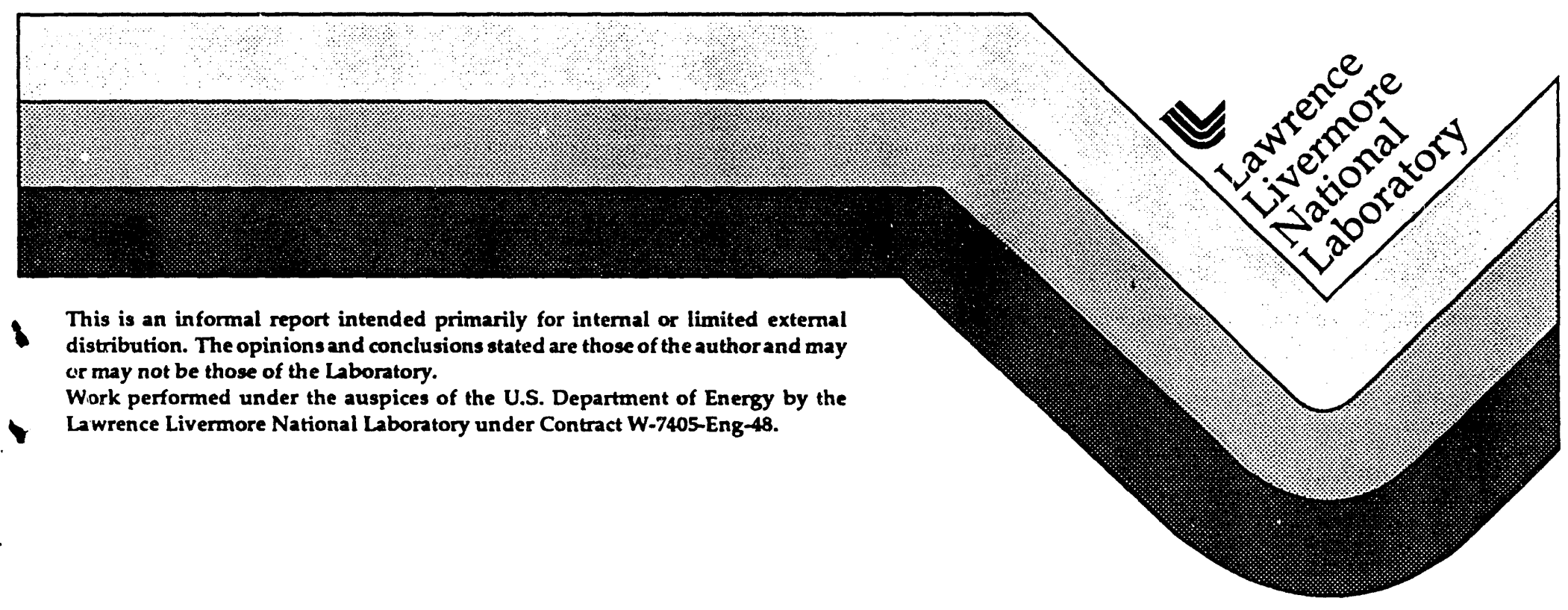




\section{DISCLAIMER}

This document was prepared as an acccount of work sponsored by an agency of the United States Government. Neither the United States Government nor the University of California nor any of their employees, makes any warranty, express or implied, or assumes any legal liability or responsibility for the accuracy, completeness, or usefulness of any information, apparatus, product, or process, disclosed, or represents that its use would not infringe privately own rights. Reference herein to any specific commercial products, process, or service by trade name, trademark, manufacturer, or otherwise, does not necessarily constitute or imply its endorsement, recommendation, or favoring by the United States Government or the University of California. The views and opinions of authors expressed herein do not necessarily state or reflect those of the United States Government or the University of California, and shall not be used for advertising or product endorsement purposes.

This report has been reproduced directly from the best available copy.

A.vailable to DOE and DOE contractors from the Office of Scientific and Technical Information P.O. Box 62, Oak Ridge, TN 37831

Prices available from (615) 576-8401, FTS 626-8401

Available to the public from the National Technical Information Service

U.S. Department of Commerce 5285 Port Royal Rd., Springfield, VA 2216 


\title{
Polarizability Corrections in Stimulated Raman Propagation rev.1
}

\author{
B. W. Shore, M. A. Johnson and S. Lowder \\ Lawrence Livermore National Laboratory \\ Livermore, CA 94550
}

\begin{abstract}
Traditional descriptions of stimulated Raman scattering relate the various Stokes and antiStokes fields to the incident pump field by means of a polarizability (tensor). This description is usable for pulsed radiation but it fails when the pump carrier frequency coincides with a resonant frequency of the medium.
\end{abstract}

We here describe a simple procedure for correcting the traditional polarizability approximation for pulse envelopes so as to account for effects of finite pump bandwidth. The correction amounts to the introduction of an auxiliary field envelope that incorporates pump dispersion.

We apply this procedure to the equations for a degenerate, Doppler broadened ensemble of three-level atoms, in which the uppermost (virtual) level is close to resonance with the pump carrier frequency. This system becomes a two-level Raman system, but with a correction to the Raman Hamiltonian and the propagation equation. The plane-wave propagation equations presented include dispersive as well as Raman effects, and allow arbitrary combinations of field polarizations.

We comment on several incidental aspects of Raman propagation, including dynamic Stark shifts, sublevel averages and fluence equations.

This work was performed under the auspices of the United States Department of Energ.' by' the Lavrence Livermore Vational Laboratory under contract no. W-7405-Eng-48. 


\section{Contents}

$\$ 1.1$ Irtrodution

\section{\$2 Definitions}

\$2.1 The Wave Equation

\$2.2 Plane-Wave Pulse Envelepe Equations

\section{\$3 The Polarization Field}

\$3.1 The Polarization Field and the Statevector

\$3.2 Resonant and Nonresonant Atoms

$\$ 3.3$ Adiabatic Elimnination with Corrections

$\$ 3.4$ Approximations

$\$ 3.5$ Alternative Viewpoints

\section{$\$ 4$ The Polarizability}

\$4.1 The Polarizability Tensor

$\$ 4.2$ Nonadiabatic Corrections

$\$ 4.3$ The Raman Hamiltonian

$\$ 4.4$ Nonadiabatic Corrections

\$4.5 Propagation: Linear and Nonlinear Parts

\$4.6 The Reference Frame

\section{\$5 Application to Three-Level Atoms}

\$5.1 The Stokes Source

\$5.2 The Anti-Stokes Source

$\$ 5.3$ The Pump Source

$\$ 5.4$ Doppler and Sublevel Averages

\$5.5 The Nonresoniant (Raman) Bloch Equations

$\S 5.6$ The Macroscopic Raman Coherences and Inversion

$\S 5.7$ Integral Equation

$\S 5.8$ The Memory Function

\$6 The Propagation Equations

\$6.1 The Maxwell-Bloch Equations

$\S 6.2$ Isotropic Medium

$\$ 6.3$ Sublevel Averages

$\$ 6.4$ Steady State Limit

\section{$\S 7$ Fluence}

$\$ 7.1$ The Fluence Equations

\$7.2 Phuion Fluences: Dirac Notation

\section{$\$ 8$ Conclusion}

$\$ 8.2$ Acknowiedgements 


\section{$\S 1.1$ Introduction}

A pulse of directed radiation (a pump beam) with well defined carrier frequency passing through a vapor (say of atoms) generates a variety of other frequencies. Some of these occur at multiples of the pump frequency (harmonics), while others occur at frequencies that are sums or differences of the pump frequency and various Bohr frequencies inherent to the medium (Raman processes).

The conventional theory of nonlinear optics characterizes all such frequency changing processes by various nonlinear susceptibilities [1]. When the pump pulse is sufficiently short and intense (relative to the response time of the medium), then traditional nonlinear optics fails, and one must describe pulse propagation by some suitable coupled version of Maxwell equations (for the field) and the time dependent Schrödinger equation (for the medium) [2].

When the pump is not resonant with any Bohr frequency, then it is possible to describe the response of the atoms to the pump by means of induced dipole moments. These act as sources for new fields at various frequencies. In special cases the induced moments can be considered independent of time, and one derives the traditional expressions for nonlinear susceptibilities. Somewhat more generally, the induced moments can be taken as those appropriate to a monochromatic pump pulse. This leads to the traditional polarizability theory of Raman processes. We present here a correction to that theory, a correction that permits more accurate description of pump bandwidth.

We are concerned specifically with the creation and propagation in an atomic vapor of a Stokes wave, i.e. a pulse whose mean carrier frequency $\omega_{S}$ is less than the mean pump carrier frequency $\omega_{p}$ by some Raman frequency $\omega_{R}$ (in turn equal to a Bohr frequency of an atom). This is an example of the stimulated Raman scattering (SRS) process [3].

In particular, we shall consider the situation in which the various pulses produce negligible change in populations, even though the atoms appreciably change the fields. 


\section{§2 Definitions}

This section defines the pulse envelope functions that we use in equations that describe propagation of pulsed radiation associated with carrier frequencies.

\section{\$2.1 The Wave Equation}

We start from the wave propagation equation for the electric field $E$ in the presence of a polarization field $\mathbf{P}$. Because our interest is with pump radiation that travels along a welldefined optical axis, defined here to be the $z$ axis, we idealize all of the fields as plane waves, i.e. fields that depend only on time $t$ and distance $z$. This approximation leads to the one-dimensional wave equation

$$
\left[\frac{\partial^{2}}{\partial z^{2}}-\frac{1}{c^{2}} \frac{\partial^{2}}{\partial t^{2}}\right] \mathbf{E}=\frac{4 \pi}{c^{2}} \frac{\partial^{2}}{\partial t^{2}} \mathbf{P}
$$

The electric field appearing here is the sum of an incident beam of laser-produced radiation (the pump field) and such other fields as may be generated by various nonlinear processes (e.g. Raman scattering, harmonic generation, etc.). The polarization field is produced by the material through which the electric field propagates -- an atomic vapor in the situation of interest. To make the mathematical description tractable we introduce a number of simplifying idealizations.

This equation forms the basis for our present analysis. Although we deal with plane waves, the results readily generalize to paraxial waves.

Much of optics treats steady illumination. For such situations it is natural to introduce temporal Fourier analysis of the fields, thereby dealing with monochromatic fields. With this approach it is customary to separate the polarization field into two parts: a linear portion that is directly proportional to the electric field (and which causes dispersion and absorption), and a nonlinear portion (which causes frequency changes). We here follow a different approach. in which we treat time dependence more explicitly. Our separation of the polarization field into two portions is based upon considerations of proximity of energy levels to resonance with carrier frequencies, but permits identification of linear and nonlinear effects.

\section{\$2.2 Plane-Wave Pulse Envelope Equations}

To avoid dealing with functions that vary rapidly in space and time (i.e. at optical frequencies) we follow conventional practice and factor out such dependence by introducing an envelope expansion. We introduce unit vectors $\mathrm{e}(\lambda)$ (in the $x, y$, plane) and envelopes $\hat{O}_{\lambda}$ and $:_{\lambda}$ (slowly varying functions of $z$ and $t$ ) for expansions with carrier frequencies $\omega_{\lambda}$ and wavevectors $k_{\lambda}$ (to be defined below)

$$
\mathrm{E}^{(+)}(z, t)=\sum_{\lambda} \frac{1}{2} \mathrm{e}(\lambda) \mathcal{S}_{\lambda} \exp \left[i k_{\lambda} z-i \omega_{\lambda} t\right]
$$




$$
\mathbf{P}^{(+)}(z, t)=\sum_{\lambda} \frac{1}{2} \mathbf{e}(\lambda) \mathscr{P}_{\lambda} \exp \left[i k_{\lambda} z-i \omega_{\lambda} t\right]
$$

The pulse intensity (power per unit area) associated with the plane-wave envelope $\mathscr{E}_{\lambda}$ is

$$
I_{\lambda}(z, t)=\frac{c}{8 \pi}\left|\mathscr{E}_{\lambda}(z, t)\right|^{2}
$$

Such expansioris have application to a variety of transient nonlinear optical processes in which an intense incident pulse (the pump wave) generates radiation at a variety of other irequencies. These frequencies may be multiples of the pump frequency (harmonic generation) or, as in the present work, they may differ from the pump frequency by discrete increments, equal to atomic Bohr frequencies. For simplicity we consider the most elementary of such processes, in which a pump pulse of frequency $\omega_{P}$ produces a Stokes pulse of frequency $\omega_{S}$, smaller than $\omega_{P}$ by the Raman frequency $\omega_{R}$. Specifically, we take the electric field and the polarization fieid to be

$$
\mathbf{E}^{(+)}(z, t)=\sum_{P} \frac{1}{2} \mathrm{e}(P) \mathcal{E}_{P} \exp \left[i k_{P} z-i \omega_{P} t\right]+\sum_{S} \frac{1}{2} \mathrm{e}(S) \mathcal{E}_{S} \exp \left[i k_{S} z-i \omega_{S} t\right]
$$

$$
\mathbf{P}^{(+)}(z, t)=\sum_{P} \frac{1}{2} \mathrm{e}(P) \mathscr{P}_{P} \exp \left[i k_{P} z-i \omega_{P} t\right]+\sum_{S} \frac{1}{2} \mathrm{e}(S) \mathscr{P}_{S} \exp \left[i k_{S} \ddot{z}-i \omega_{S} t\right]
$$

$$
\omega_{P}=\text { pump frequency, } \quad \omega_{S}=\omega_{P}-\omega_{R}=\text { Stokes frequency }
$$

where the sums go over (two) directions of the unit vectors $e(\lambda)$ that define the instantaneous polarization direction. Each of the components of this sum can act upon the atoms to produce additional frequencies. We consider only the simplest situation, in which only the pump field is sufficiently intense to induce appreciable distortion of the atoms. A more complete description of Raman processes must include a succession of Stokes and antiStokes frequencies.

We make the traditional slowly varying envelope approximation (SVEA), in which we neglect second derivatives. For notational convenience we define a carrier index $\eta_{\lambda}$ such that

$$
c k_{\lambda}=\eta_{\lambda} \omega_{\lambda}
$$

the envelope equation becomes

$$
\left[\frac{\partial}{\partial z}+\frac{1}{c \eta_{\lambda}} \frac{\partial}{\partial t}-\frac{i \omega_{\lambda}}{2 c \eta_{\lambda}}\left[1-\left(\eta_{\lambda}\right)^{2}\right]\right] \mathcal{G}_{\lambda}=i \frac{2 \pi \omega_{\lambda}}{c \eta_{\lambda}}\left[1+\frac{2 i}{\omega_{\lambda}} \frac{\partial}{\partial t}\right] \mathcal{P}_{\lambda} .
$$


We omit the time derivative of $\mathscr{P}_{\lambda}$ as being slow compared with an optical cycle. To proceed we must specify the polarization envelope $\mathscr{P}_{\lambda}$ and we must specify $\eta_{\lambda}--$ that is, we must specify the carrier wavelength that we associate with carrier frequency $\omega_{\lambda}$. Two choices are used in practice: either set $\eta_{\lambda}$ equal to the refractive index (the traditional choice), or set $\eta_{\lambda}$ to unity (our choice). We choose a coordinate systern that moves with speed $c$, so that in this moving window the equation becomes simply

$$
\frac{\partial}{\partial z} \mathscr{E}_{\lambda}(z, t)=i \frac{2 \pi \omega_{\lambda}}{c} \mathscr{P}_{\lambda}(z, t)
$$

This is the basic propagation equation for plane-wave pulses whose envelopes vary slowly in time and space (compared with carrier frequency and wavelength). The polarization field is responsible for all deviations from distortionless propagation in this moving frame: it accounts for linear effects, such as dispersion and group velocity, as well as nonlinear effects, such as change of frequency (e.g. Raman processes or harmonic generation). 


\section{$\S 3$ The Polarization Field}

This section connects the polarization field, the source of Raman processes, to an average of atomic properties deducible from an atomic statevector. We follow the conventional introduction of an induced dipole moment, leading to a polarizability, but we include corrections to that approximation.

\section{§3.1 The Polarization Field and the Statevector}

The polarization field $\mathbf{P}$ (with or without further separation into linear and nonlinear or resonant and nonresonant parts) is to be determined by evaluating the distribution of atomic dipole moments. For a single species of atom, distributed with number density $\mathcal{N}$, the connection is

$$
\mathbf{P}(z, t)=\mathcal{N}(z, t)\langle\mathbf{d}(z, t)\rangle=\mathcal{N}(z, t)\langle\Psi(z, t)|\mathbf{d}| \Psi(z, t)\rangle
$$

where $\Psi(z, t)$ is the time dependent atomic statevector for an atom whose center of mass is at $z$. More generally we must consider not just one species of atom but many. We must consider both resonant and nonresonant atoms and, for each, we must consider a distribution of Doppler velocities and orientations. The general form of the construction is

$$
\mathbf{P}=\sum_{\alpha} \mathcal{N}_{\alpha}\langle\mathbf{d}\rangle_{\alpha}
$$

where $\alpha$ labels a set of atomic attributes (i.e. various isotopes, various Doppler velocities, etc.).

By comparing the atomic expansion for $\mathbf{P}$ with the assumed envelope expansion for $\mathbf{P}$ we find the connection between the envelope $\mathscr{P}_{\lambda}$ and the positive-frequency part of the dipole-moment expectation value:

$$
\mathscr{P}_{\lambda}=2 \sum_{\alpha} \mathcal{N}_{\alpha} \mathrm{e}(\lambda)^{*} \cdot\left\langle\mathrm{d}^{(+)}\right\rangle_{\alpha} \exp \left(-i k_{\lambda} z+i \omega_{\lambda} l\right)
$$

That is, the slowly varying envelope $\mathscr{P}_{\lambda}$ is the portion of the macroscopic dipole moment that varies as $\exp \left(+i k_{\lambda} z-i \omega_{\lambda} t\right)$. This is the envelope function for the entire polarization field (i.e. linear and nonlinear, resonant and nonresonant).

When dealing with pure states we express the atomic statevector $\Psi(z, \ell)$ at position $=$ and time $t$ as a superposition of atomic basis states $\psi_{a}$ with phases $\zeta_{a}(z, t)=W_{a} t-K_{q} z$ (to be defined below)

$$
\Psi(z, t)=\sum_{a} \psi_{a} C_{a}(z, t) \exp \left[i K_{a} z-i W_{a} t\right]
$$


The dipole moment of a pure atomic state is evaluated by considering all dipole matrix elements between basis states, taken with appropriate phases:

$$
\langle\Psi(z, t)|\mathrm{d}| \Psi(z, t)\rangle=\sum_{a b} C_{a}(t)^{*} C_{b}(t)\langle a|\mathrm{~d}| b\rangle \exp \left[-i\left(\zeta_{b}-\zeta_{a}\right)\right]
$$

We define the (pure-state) atomic density matrix $\rho$ to have the elements

$$
\rho_{a b}(z, t)=C_{a}(z, t) C_{b}(z, t)^{*} .
$$

Then the polarization field from a single species of atom has the expression

$$
\mathbf{P}(z, t)=\mathcal{N}\langle\mathbf{d}\rangle=\mathcal{N} \sum_{a b}\langle a|\mathbf{d}| b\rangle \rho_{b a}(z, t) \exp \left[i\left(K_{b}-K_{a}\right) z-i\left(W_{b}-W_{a}\right) t\right]
$$

The positive frequency part of this field is the contribution for which $W_{a}<W_{b}$,

$$
\mathbf{P}(z, t)^{(+)}=\mathcal{N} \sum_{a<b}\langle a|\mathrm{~d}| b\rangle \rho_{b a}(z, t) \exp \left[i\left(K_{b}-K_{a}\right) z-i\left(W_{b}-W_{a}\right) t\right]
$$

It follows that the pulse envelope is the sum

$$
\mathscr{P}_{\lambda}(z, t)=2 \mathcal{N} \sum_{a<b} d_{a b}(\lambda) \rho_{b a}(z, t) \exp \left[i\left(K_{b}-K_{a}-k_{\lambda}\right) z-i\left(W_{b}-W_{a}-\omega_{\lambda}\right) t\right]
$$

where

$$
d_{a b}(\lambda)=\mathrm{e}(\lambda)^{*} \cdot\langle a|\mathrm{~d}| b\rangle
$$

is a component of a dipole transition moment.

As noted above, the atomic phases (embodied in the quantities $K_{a}$ and $W_{a}$ ) are arbitrary. Physical observables do not depend on the choice of these phases, although the phases do appear in the various equations of motion. They will be chosen below for convenience in extracting the pump and Stokes wave contributions. Specifically, they will be chosen to remove from the density matrix variations at optical frequencies and optical wavelengths. When this is done. we can identify the various pairs of frequency differences $W_{a}-W_{b}$ as the frequencies of the several carrier waves that contribute to the polarization field; the coefficients of such exponentials are the polarization-ficld envelopes $\mathscr{P}_{\lambda}$.

The introduction of $\rho_{a b}$ in place of $\Psi$ permits generalization to mixed-state situations and allows the introduction of homogeneous relaxation (e.g., spontaneous emission). We need to do two things: 
1. From an appropriate atomic equation of motion (a suitable generalization of the time-dependent Schrödinger equation) we determine $\rho_{a b}(z, t)$

2. We separate the portion of this sum that varies with the Stokes carrier frequency. This provides the source $\mathscr{P}_{S}$ for Stokes radiation

\section{$\S$ The Time Dependent Schrödinger Equation}

The time dependent Schrödinger equation for the statevector $\Psi(z, t)$ appropriate to a single atom whose center of mass is at $z$,

$$
\hbar \frac{\partial}{\partial t} \Psi(z, t)=-i H(z, t) \Psi(z, t)
$$

taken with the expansion

$$
\Psi(z, t)=\sum_{a} \psi_{a} C_{a}(z, t) \exp \left[-i \zeta_{a}\right]
$$

leads to the following equations for the probability amplitudes

$$
\begin{aligned}
& \hbar \frac{\partial}{\partial t} C_{n}(z, t)=-i\left[H_{n n}-\hbar \dot{\zeta}_{n}\right] C_{n}(z, t) \\
& \quad-i \sum_{m \neq n} H_{n m}(z, t) \exp \left[i\left(\zeta_{n}-\zeta_{m}\right)\right] C_{m}(z, t)
\end{aligned}
$$

\section{$\S 3.2$ Resonant and Nonresonant Atoms}

Let us consider separately the contributions from two types of atoms: those that have siugle-photon dipole transitions in near resonance with the field (resonant atoms), and those that do not (nonresonant atoms). For the latter atoms the contribution to the polarization field comes from induced dipole moments. With this separation the polarization envelope has two contributions,

$$
\mathscr{P}_{\lambda}=\operatorname{res} \mathscr{P}_{\lambda}+\operatorname{nr} \mathscr{P}_{\lambda} \text {. }
$$

For a single species of resonant or nonresonant atoms the polarization field is

$$
\mathbf{P}={ }^{r e s}, \mathcal{V}\langle\mathrm{d}\rangle_{r e s}+n{ }_{-}-V^{r}\langle\mathbf{d}\rangle_{n r}
$$

To separate these two classes of transitions we separate the atomic basis states into two classes. In the first set we place the ground state and those states that connect with the ground state either by resonant dipole interaction or by various Raman processes. Let us call this set, the states $\psi_{p}$, the $P$ set. The remaining states form the $Q$ set, and are denoted $\psi_{q}$. They are each orthogonal to each of the $P$ states. These states have the properties 


$$
H^{0} \psi_{p}=E_{p} \psi_{p}, \quad \quad H^{0} \psi_{q}=E_{q} \psi_{q}, \quad\left\langle\psi_{p} \mid \psi_{q}\right\rangle=0
$$

Here $E_{p}$ is the energy eigenvalue of atomic state $p$ in the absence of the electric field $\mathrm{E}(t)$. With the separation of basis states in this manner we can express the atomic state vector $\Psi(t)$ at all times as a superposition of the form

$$
\Psi(t)=\sum_{p} \psi_{p} C_{p}(t) \exp \left(-i \zeta_{p}\right)+\sum_{q} \psi_{q} C_{q}(t) \exp \left(-i \zeta_{q}\right) .
$$

The time variable $t$ has, in the present context of the TD Schrödinger equation, an arbitrary starting point. In the larger context of propagation, it is the time relative to the arrival of a fiducial pulse of radiation at the location of the atom.

\section{The Dirac Picture}

The choice of phase $\zeta_{p}$ is arbitrary. We here introduce the Dirac picture by choosing the time variation of the phase to be the unperturbed atomic eigenvalue,

$$
\zeta_{p}(z, t)=W_{p} t-K_{p} z, \quad \hbar W_{p}=E_{p}
$$

\section{\$3.3 Adiabatic Elimination with Corrections}

Atomic states that are not connected with the atomic ground state by near resonant transitions undergo little change with time, and their influence can be incorporated into various induced mornents by means of adiabatic elimination. This procedure, the elimination of amplitude $C_{q}(t)$ for a virtual state, is based upon substituting the mode expansion for the field (at fixed position),

$$
\left.\mathrm{E}(t)=\sum_{\lambda} \int_{0} d \omega^{\prime} \exp \left(-i \omega^{\prime} t-\omega_{\lambda} t\right) \tilde{\mathrm{E}}_{\lambda} i \omega^{\prime}\right)+\sum_{\lambda} \int_{0} d \omega^{\prime} \exp \left(+i \omega^{\prime} t+\omega_{\lambda} t\right) \tilde{\mathbf{E}}_{\lambda}\left(\omega^{\prime}\right)^{*}
$$

into the time-dependent Schrödinger equation (with electric dipole interaction) for the virtual-state amplitude $C_{q}(t)$

$$
\hbar \frac{d}{d t} C_{q}(t)=+i \sum_{p}\langle q|\mathrm{~d} \cdot \mathrm{E}(t)| p\rangle C_{p}(t) \exp \left[-i\left(W_{p}-W_{q}\right) t\right]
$$

The (exact) solution, before making the adiabatic approximation, involves a double Fourier integral in which integrands become linked through denominators $W_{q}-W_{p}+\omega_{1} \pm \omega_{2} \pm \omega_{\lambda}$ :

$$
\hbar C_{q}(i)=-\sum_{p \lambda} \int d \omega_{1} \int d \omega_{2} \frac{\exp \left[-i\left(W_{p}-W_{q}+\omega_{1}+\omega_{2}+\omega_{\lambda}\right) t\right]}{W_{p}-W_{q}+\omega_{1}+\omega_{2}+\omega_{\lambda}}\left\langle q\left|\mathrm{~d} \cdot \tilde{\mathrm{E}}_{\lambda}\left(\omega_{2}\right)\right| p\right\rangle \tilde{C}_{p}\left(\omega_{1}\right)
$$




$$
-\sum_{p \lambda} \int d \omega_{1} \int d \omega_{2} \frac{\exp \left[-i\left(W_{p}-W_{q}+\omega_{1}+\omega_{2}-\omega_{\lambda}\right) t\right]}{W_{p}-W_{q}+\omega_{1}+\omega_{2}-\omega_{\lambda}}\left\langle q\left|\mathbf{d} \cdot \tilde{\mathbf{E}}_{\lambda}\left(\omega_{2}\right)^{*}\right| p\right\rangle \tilde{C}_{p}\left(\omega_{1}\right)
$$

The first of these sums is known as the resonant contribution (it has a vanishing denominator when the pump frequency equals a Bohr frequency), while the second is the anti-resonant contribution. To disentangle this coupling between field and atom we expand the denominator of the integral:

$$
\frac{1}{W_{q}-W_{p}-\omega_{1} \pm \omega_{\lambda} \pm \omega_{2}}=\sum_{k==^{\prime}}^{\infty} \frac{\left(\omega_{1} \pm \omega_{2}\right)^{k}}{\left(W_{q}-W_{p} \pm \omega_{\lambda}\right)^{k+1}}
$$

Then we separate the powers of $\omega_{1}$ and $\omega_{2}$ (the integration variables) by using the binomial expansion. For the resonant sum we use the formula

$$
\frac{1}{W_{q}-W_{p}-\omega_{\lambda}-\omega_{1}-\omega_{2}}=\frac{1}{\Delta_{q p \lambda}} \sum_{k m}(k)\left(\frac{\omega_{1}}{n}\right) m\left(\frac{\omega_{2}}{\Delta_{q p \lambda}}\right)^{k-1 n}
$$

Here $\Delta_{q p \lambda}$ is the detuning of the carrier frequency $\omega_{\lambda}$ from the $p, q$ Bohr frequency:

$$
\Delta_{q p \lambda} \equiv W_{q}-W_{p}-\omega_{\lambda}
$$

This expansion allows us to write the resonant contribu* on to the virtual-state amplitude as

$$
\begin{aligned}
\hbar C_{q}(t)=+\frac{1}{2} & \sum_{p \lambda} \exp \left[i \Delta_{q p \lambda} t\right] \frac{\langle q|\mathrm{~d} \cdot \mathrm{e}(\lambda)| p\rangle}{\Delta_{q p \lambda}} \sum_{k m}\left(\begin{array}{c}
k \\
m
\end{array}\right) \\
& \times \int d \omega_{1} \exp \left[-i \omega_{1} t\right]\left(\frac{\omega_{1}}{\Delta_{q p \lambda}}\right) m \mathfrak{C}_{\lambda}\left(\omega_{1}\right) \int d \omega_{2} \exp \left[-i \omega_{2} t\right]\left(\frac{\omega_{2}}{\Delta_{q p \lambda}}\right) k-m \tilde{C}_{p}\left(\omega_{2}\right)
\end{aligned}
$$

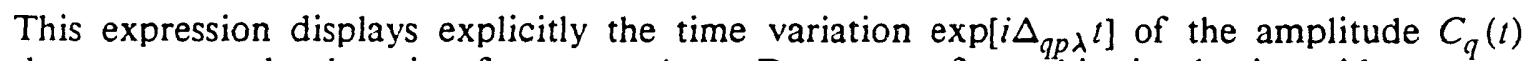
that occurs at the detuning frequency $\Delta_{q p \lambda}$. Departures from this simple sinisoid are produced by the terms of the series.

Evidently what matters is the range of frequencies that contribute to the Fourier amplitudes (i.e. the bandwidths) compared with the detuning $\Delta_{q p \lambda}$. When the carrier frequency is sufficiently far from resonance the usual polarizability approximation applies (and one may require both resonant and anti-resonant contributions). Our concern is with near resonant excitation, for which the anti-resonant contribution is negligible but the field bandwidth is not neccessarily small. 


\section{§3.4 Approximations}

The frequencies $\omega_{1}$ and $\omega_{2}$ used in the Fourier integrals are measured relative to central frequencies that are intended to reproduce the dominant high frequency component of the variable. For the field this is the carrier frequency, while for the atom it is a Bohr frequency (we deal here with the $P$ states, for which detuning is negligible). When the Fourier amplitudes are sharply peaked in frequency about the values $\omega_{1}=0$ and $\omega_{2}=0$ we need retain only the first term of the series:

$$
\hbar C_{q}(t)=+\frac{1}{2} \sum_{p \lambda} \exp \left[i \Delta_{q p \lambda} t\right] \frac{\langle q|\mathrm{~d} \cdot \mathrm{e}(\lambda)| p\rangle}{\Delta_{q p \lambda}} \mathscr{E}_{\lambda}(t) C_{p}(t)
$$

This leads to the conventional polarizability approximation (see below), in which the virtual-state amplitude at time $t$ is directly proportional to the field at that same time. More generally we can convert the separate Fourier integrals into time derivatives:

$$
\begin{aligned}
\hbar C_{q}(t) & =+\frac{2}{2} \sum_{p \lambda} \exp \left[i \Delta_{q p \lambda} t\right] \frac{\langle q|\mathrm{~d} \cdot \mathrm{e}(\lambda)| p\rangle}{\Delta_{q p \lambda}} \sum_{k m}\left(\begin{array}{c}
k \\
m
\end{array}\right] \\
& \times\left[\left(\frac{i}{\Delta_{q p \lambda}} \frac{d}{d t}\right)^{m} \mathcal{E}_{\lambda}(t)\right]\left[\left(\frac{i}{\Delta_{q p \lambda}} \frac{d}{d t}\right)^{k-m} C_{p}(t)\right] .
\end{aligned}
$$

This (exact) expression shows that the important time variations are those whose time scale is short compared with the inverse of the carrier detuning. Although the relationship between $C_{q}(t)$ and $C_{p}(t)$ is complicated by various derivatives, it is still a linear relationship, and can still be written in the form

$$
\hbar C_{q}(t)=\sum_{p \lambda} M_{q p}^{\lambda}(t) C_{p}(t)
$$

The exact connection between $C_{q}(t)$ and $C_{p}(t)$ requires time derivatives of both the field and the atomic state. For Raman excitation we typically consider situations in which the initial states (the $P$ states) remain relatively unchanged in time, while the virtual states acquire time dependence from the excitation. In this traditional approximation, of slowly varying populations, we neglect the derivatives of the atomic amplitude, and the equation simplifies to

$\hbar C_{q}(t)=+\frac{1}{2} \sum_{p \lambda} \exp \left[i \Delta_{q p \lambda} l\right] \frac{\langle q|\mathrm{~d} \cdot \mathrm{e}(\lambda)| p\rangle}{\Delta_{q p \lambda}} C_{p}(t) \sum_{k=0}^{\infty}\left(\frac{i}{\Delta_{q p \lambda}} \frac{d}{d t}\right) k \mathcal{E}_{\lambda}(t)$.

When a single virtual state dominates the induced dipole moment it is useful to regard the infinite summation of derivatives as producing a modified field, 


$$
\mathscr{F}_{\lambda}(t)=\left[1+\frac{i}{\Delta_{q p \lambda}} \frac{d}{d t}+\ldots\right] \mathscr{E}_{\lambda}(t)=\sum_{k}\left(\frac{i}{\Delta_{q p \lambda}} \frac{d}{d t}\right) k \mathscr{E}_{\lambda}(t)
$$

or

$$
\mathscr{F}_{\lambda}(t)=\sum_{k} \int d \omega_{1} \exp \left[-i \omega_{1} t\right]\left(\frac{\omega_{1}}{\Delta_{q p \lambda}}\right) k \mathscr{J}_{\lambda}\left(\omega_{1}\right)
$$

That is, we can write the matrix connecting $C_{q}(t)$ with $C_{p}(t)$ as

$$
M_{q p}^{\lambda}(t)=\frac{1}{2} \exp \left[-i\left(W_{p}-W_{q}+\omega_{\lambda}\right) t\right] \frac{\langle q|\mathrm{~d} \cdot \mathrm{e}(\lambda)| p\rangle}{W_{q}-W_{p}-\omega_{\lambda}} \mathscr{F}_{\lambda}(t)
$$

where now $\mathscr{F}_{\lambda}(t)$ appears in place of $\mathscr{E}_{\lambda}(t)$. When the frequency content of the envelope is restricted to values less than the detuning then the series can be summed to produce the formula

$$
\mathscr{F}_{\lambda}(t)=\int d \omega_{1} \frac{\exp \left[-i \omega_{1} t\right]}{1-\left(\omega_{1} / \Delta_{q p \lambda}\right)} \mathcal{O}_{\lambda}\left(\omega_{1}\right)
$$

The failure of this series to converge for values of $\omega_{1}$ exceeding the detuning $\Delta$ expresses the breakdown of our assumption that population remains primarily in the $P$ states. When resonant frequencies are present then Rabi oscillations occur into the $Q$ states, and the procedure of adiabatic elimination fails.

The radius of convergence of the series may be extenaed by including spontaneous emission loss terms in the atomic Hamiltonian $H^{0}$ as non-Hermitian elements.

Rather than exhibit an explicit dependence upon a particular virtual state, it is of ten useful to introiuce derivatives with respect to carrier frequency $\omega_{\lambda}$, using the property

$$
\frac{1}{\left(\Delta_{q p \lambda}\right)^{k}}=\left(\frac{d}{d \omega_{\lambda}}\right)^{k}
$$

and rewrite Eqn. (3.4-2) as

$\hbar C_{q}(t)=+\frac{1}{2} \sum_{p \lambda} \exp \left[i \Delta_{q p \lambda} t\right] C_{p}(t) \sum_{k=0}^{\infty} \frac{1}{k !}\left(\frac{d}{d \omega_{\lambda}}\right) k \frac{\langle q|\mathbf{d} \cdot \mathbf{e}(\lambda)| p\rangle}{\Delta_{q p \lambda}}\left(i \frac{d}{d t}\right) k \mathcal{E}_{\lambda}(t)$

With the understanding that frequency derivatives are to be applied to induced moments, we can use this result to define the field $\mathcal{F}_{\lambda}(t)$ as 


$$
\mathscr{F}_{\lambda}(t)=\sum_{k=0}^{\infty} \frac{1}{k !}\left(\frac{d}{d \omega_{\lambda}}\right)^{k}\left(i \frac{d}{d t}\right)^{k} \mathscr{E}_{\lambda}(t)
$$

\section{$\S 3.5$ Alternative Viewpoints}

The preceding result may be viewed in alternative ways. In the approximation of slowly changing atomic amplitudes we have the equation

$$
\begin{aligned}
\hbar C_{q}(t) & =-\sum_{p \lambda} C_{p}(t) \int_{\cdot} d \omega^{\prime} \frac{\exp \left[-i\left(\omega^{\prime}-\Delta_{q p \lambda}\right) t\right]}{\omega^{\prime}-\Delta_{q p \lambda}}\left\langle q\left|\mathbf{d} \cdot \tilde{\mathbf{E}}_{\lambda}\left(\omega^{\prime}\right)\right| p\right\rangle \\
& -\sum_{p \lambda} C_{p}(t) \int_{\cdot} d \omega^{\prime} \frac{\exp \left[-i\left(\omega^{\prime}-\Delta_{q p \lambda}+2 \omega_{\lambda}\right) t\right]}{\omega^{\prime}-\Delta_{q p \lambda}+2 \omega_{\lambda}}\left\langle q\left|\mathbf{d} \cdot \tilde{\mathbf{E}}_{\lambda}\left(\omega^{\prime}\right)^{*}\right| p\right\rangle
\end{aligned}
$$

With the omission of the nonresonant term this formula becomes

$\hbar C_{q}(t)=-\sum_{p \lambda} C_{p}(t) \frac{\exp \left[+i \Delta_{q p \lambda} t\right]}{\Delta_{q p \lambda}} \int d \omega^{\prime} \exp \left[-i \omega^{\prime} t\right] \frac{\left\langle q\left|\mathbf{d} \cdot \tilde{\mathbf{E}}_{\lambda}\left(\omega^{\prime}\right)\right| p\right\rangle}{\left(\omega^{\prime} / \Delta_{q p \lambda}\right)-1}$

The integrand of the Fourier transform may be regarded in two ways: either as the product of a frequency dependent induced dipole moment times the electric field transform,

$$
\frac{\left\langle q\left|\mathbf{d} \cdot \tilde{\mathbf{E}}_{\lambda}(\omega)\right| p\right\rangle}{\left(\omega / \Delta_{q p \lambda}\right)-1}=\left\langle q\left|\mathbf{d}(\omega) \cdot \tilde{E}_{\lambda}(\omega)\right| \mathbf{p}\right\rangle, \quad \mathbf{d}(\omega)=\frac{\mathbf{d}}{\left(\omega / \Delta_{q p \lambda}\right)-1}
$$

or else as the product of the ordinary dipole moment with a modified field:

$$
\frac{\left\langle q\left|\mathbf{d} \cdot \tilde{\mathbf{E}}_{\lambda}(\omega)\right| p\right\rangle}{\left(\omega / \Delta_{q p \lambda}\right)-1}=\left\langle q\left|\mathbf{d} \cdot \tilde{\mathbf{F}}_{\lambda}(\omega)\right| p\right\rangle, \quad \tilde{\mathbf{F}}_{\lambda}(\omega)=\frac{\tilde{\mathbf{E}}_{\lambda}(\omega)}{\left(\omega / \Delta_{q p \lambda}\right)-1}
$$

This modification applies wherever the induced dipole moment appears in formulas.

Although these results permit the introduction of arbitrary bandwidth for the pump field, they are limited by the requirement that the amplitude of the virtual state be slowly varying. Thus they break down as the pump comes closer to resonance and the virtual populations become appreciable. 


\section{\$4 The Polarizability}

We here define a poiarizability tensor operator (responsible for the induced dipole moment), and a corresponding tensor that incorporates the polarization directions of pairs of fields. These appear in the construction of the polarization envelope and in the Raman Hamiltonian that governs the time evolution of the density matrix. We show the corrections that are required to both the polarization field and the Raman Hamiltonian when bandwidths become appreciable. We exhibit the propagation equation, after separating linear and nonlinear contributions to the polarizability; the former are responsible for dispersion, the latter produce Raman scattering.

\section{\$4.1 The Polarizability Tensor}

To identify the envelope source $\mathscr{P}_{\lambda}$ we compare the expansion for $\mathbf{P}$, involving the phase $\exp \left(-i \omega_{\lambda} t\right)$, with the expansion for $\langle\mathrm{d}\rangle$, involving the phases $\exp \left[-i\left(W_{p}-W_{p^{\prime}}+\omega_{\lambda}\right) t\right]$. We require that the two phases be equal and that the two vector directions be equal. The result is the single-species formula

$$
\begin{aligned}
{ }^{n r} \mathscr{P}_{\lambda^{\prime}}(z, t)= & \sum_{i j p p^{\prime} \lambda} n r \mathcal{N}{ }^{n r} \rho_{p p^{\prime}} \in\left(\lambda^{\prime}, \lambda\right)_{i j}\left\langle p^{\prime}\left|X_{i j}\left(\omega_{\lambda}\right)\right| p\right\rangle \mathcal{E}_{\lambda}(z, t) \\
& \times \exp \left[i\left(K_{p}-K_{p^{\prime}}+k_{\lambda}-k_{\lambda^{\prime}}\right) z\right] \exp \left[-i\left(W_{p}-W_{p^{\prime}}+\omega_{\lambda}-\omega_{\lambda^{\prime}}\right) t\right]
\end{aligned}
$$

for the contribution to the polarization field by nonresonant atoms. (This expression includes both linear and nonlinear contributions.) The phase factors $W_{p}-W_{p^{\prime}}+\omega_{\lambda}$ provide the frequencies of the various carrier waves that constitute the polarization field. The polarization properties (i.e. field direction) of the electric field are embodied in the unit vectors $\mathbf{e}(\lambda)$ and in the tensors

$$
\epsilon_{i j}\left(\lambda^{\prime}, \lambda\right)=e_{i}\left(\lambda^{\prime}\right)^{*} e_{j}(\lambda), \quad \in\left(\lambda^{\prime}, \lambda\right)=\mathbf{e}\left(\lambda^{\prime}\right)^{*} \mathbf{e}(\lambda) .
$$

The atomic polarizibility tensor operator $\mathbf{X}(\omega)$ appearing here has matrix elements

$$
\left\langle p^{\prime}\left|X_{i j}(\omega)\right| p\right\rangle=\sum_{q}\left[\frac{\left\langle p^{\prime}\left|d_{i}\right| q\right\rangle\left\langle q\left|d_{j}\right| p\right\rangle}{\hbar\left(W_{q}-W_{p}-\omega\right)}+\frac{\left\langle p^{\prime}\left|d_{j}\right| q\right\rangle\left\langle q\left|d_{i}\right| p\right\rangle}{\hbar\left(W_{q}-W_{p^{\prime}}+\omega\right)}\right] .
$$

Note that the indices $i j$ are interchanged in the second summation, and that the first sum contains the initial-state energy $E_{p}=\hbar I V_{p}$ whereas the second sum contains the final-state energy $E_{p^{\prime}}=\hbar W_{p^{\prime}}$. The first sum (the resonant contribution) dominates when the frequency $\omega$ is close to a Bohr frequency. The second sum (the antiresonant contribution) must be retained when $\omega$ is not close to any resonance. These matrix elements have the property

$$
\left\langle p^{\prime}\left|X_{i j}(\omega)\right| p\right\rangle=\left\langle p\left|X_{i j}(-\omega)\right| p^{\prime}\right\rangle^{*} .
$$

It is customary to define the polarizability as the proportionality between the single-atom induced dipole moment and the electric field. We extend that definition to the more gen- 
eral polarizability

$$
\begin{aligned}
& \alpha_{p^{\prime} p}\left(\lambda^{\prime}, \lambda\right)=\sum_{i j} \in\left(\lambda^{\prime}, \lambda\right)_{i j}\left\langle p^{\prime}\left|X_{i j}\left(\omega_{\lambda}\right)\right| p\right\rangle \\
& =\in\left(\lambda^{\prime}, Y\right):\left\langle p^{\prime}\left|\mathbf{X}\left(\omega_{\lambda}\right)\right| p\right\rangle \equiv \mathbf{e}(\lambda)^{*} \cdot\left\langle p^{\prime}\left|\mathbf{X}\left(\omega_{\lambda}\right)\right| p\right\rangle \cdot e(\lambda) . \\
& =\sum_{K Q}(-1)^{K+Q} \in\left(\lambda^{\prime}, \lambda\right)_{-Q}^{\{K} \mid\left\langle p^{\prime}(\mathbf{X}) \omega_{\lambda} Q^{(K)} \mid p\right\rangle
\end{aligned}
$$

This polarizability depends generally upon two atomic states (indices $p, p^{\prime}$ ) and upon two field directions (indices $\lambda, \lambda^{\prime}$ ) as well as a carrier frequency $\omega_{\lambda}$.

\section{\$4.2 Nonadiabatic Corrections}

As discussed previously (section $\$ 3.7$ ) the effects of broadband radiation can be taken into account by replacing the field responsible for an induced moment by the filtered field $\mathscr{F}_{\lambda}(t)$ defined above. With the polarizability correction $w^{\prime} \cdot$ obtain the formula

$$
\begin{aligned}
\operatorname{nr}_{\mathcal{P}_{\lambda^{\prime}}(z, t)=} & \sum_{p p^{\prime} \lambda} n r \operatorname{rr}{ }^{n r} \rho_{p p^{\prime}} \alpha_{p^{\prime} p}\left(\lambda^{\prime}, \lambda\right) \mathscr{F}_{\lambda}(z, t) \\
& \times \exp \left[i\left(K_{p}-K_{p^{\prime}}+k_{\lambda}-k_{\lambda^{\prime}}\right) z\right] \exp \left[-i\left(W_{p}-W_{p^{\prime}}+\omega_{\lambda}-\omega_{\lambda^{\prime}}\right) t\right] .
\end{aligned}
$$

The exponential time-dependent phase factor acts to select only those terms in the sum for which the field carrier frequencies differ by the atomic frequency value $W_{p}-W_{p^{\prime}}$. This constraint still leaves a sum over polarization directions of the field.

To evaluate this expression for the polarization envelope associated with a given field it is neccessary to evaluate, in some approximation, the elements of the density matrix. For this purpose it is neccessary to obtain, and solve, equations of atomic dynamics. In the simplest case one takes the diagonal elements of the density matrix to be stationary quantities (the populations), and obtains an expression for the off-diagonal elements in terms of these. A subsequent section applies this approach.

\section{$\S 4.3$ The Raman Hamiltonian}

The polarization envelope $\mathscr{P}_{\lambda}$ for a Raman process involves the product of two atomic quantities: a (frequency dependent) polarizability, and the elements of a density matrix. The latter is to be determined by solving the (generalized Bloch) equation in the rotating wave approximation,

$$
\frac{d}{d t} \rho=i[W, \rho]-\Gamma \rho+h
$$


Hers the $W$ is the effective Raman Hamiltonian, the operator $\Gamma$ provides homogeneous relaxation and the operator $h$ is a Langevin noise source needed to preserve proper quantum properties in the presence of the relaxation term $\Gamma$.

The general procedure used to derive the Raman Hamiltonian is as follows. We first separate complete set of atomic states into two classes: those linked by resonant one- or two-photon transitions ( $F$ states), and those that are nonresonant ( $Q$ states). We expand the atomic state vector $\Psi(z, t)$ for an atom centered at location $z$ as

$$
\begin{aligned}
\Psi(z, t) & =\sum_{p} \psi_{p} C_{p}(z, t) \exp \left[i\left(K_{p} z-W_{p} t\right)\right] \\
& +\sum_{q} \psi_{q} C_{q}(z, t) \exp \left[i\left(K_{q} z-W_{q} t\right)\right]
\end{aligned}
$$

The phases $K_{p} z, W_{p} t$ and $W_{q} t$ remain to be specified. The most appropriate choice for purposes cf adiabatic elimination is that of the Dirac picture: we take $\hbar W_{p}$ to be the unperturbed energy eigenvalue of state $p$. This choice, which we follow here, removes from the effective Hamiltonian the diagonal terms that are independent of the interaction. (An alternative choice is to incorporate into $\hbar W_{p}$ the dynamic Stark shifts.)

We next adiabatically eliminate $Q$ states from time-dependent Schrödinger equation (or Bloch equations). The resulting effective RWA Hamiltonian comprises two parts:

$$
W_{p^{\prime} p}=W_{p p}^{d i p}+W_{p^{\prime} p}^{r a m} .
$$

The direct dipole part is

$$
\begin{aligned}
& W_{p p}^{d i p}=-\frac{1}{2 \hbar} \sum_{\lambda}\left\langle p^{\circ}|\mathbf{d}| p\right\rangle \cdot \mathbf{e}(\lambda) \mathcal{E}_{\lambda} \exp \left[i\left(W_{p^{\prime}}-W_{p}-\omega_{\lambda}\right) t\right] \exp \left[-i\left(K_{p^{\prime}}-K_{p}-k_{\lambda}\right) z\right] \\
& -\frac{1}{2 \hbar} \sum_{\lambda}\left\langle p^{\prime}|\mathbf{d}| p\right\rangle \cdot \mathbf{e}(\lambda)^{*} \mathcal{E}_{\lambda}^{*} \exp \left[i\left(W_{p^{\prime}}-W_{p}+\omega_{\lambda}\right) t\right] \exp \left[-i\left(K_{p^{\prime}}-K_{p}+k_{\lambda}\right) z\right]
\end{aligned}
$$

The Raman part incorporates all indirect transitions to $Q$ states. In the traditional polarizability approximation it is

$$
W_{p^{\prime} p}^{r a m}=-\frac{1}{4 \hbar} \sum_{\lambda \lambda^{\prime}}\left\langle p^{\prime}\left|\mathbf{X}\left(\omega_{\lambda}\right)\right| p\right\rangle: \in\left(\lambda^{\prime}, \lambda\right) \cdot \mathscr{E}_{\lambda^{\prime}}{ }^{*} \mathscr{G}_{\lambda}
$$




$$
\times \exp \left[i\left(W_{p^{\prime}}-W_{p}+\omega_{\lambda^{\prime}}-\omega_{\lambda}\right) t\right] \exp \left[-i\left(K_{p^{\prime}}-K_{p}+k_{\lambda^{\prime}}-k_{\lambda}\right) z\right] .
$$

The electric field polarization tensor $\in\left(\lambda^{\prime}, \lambda\right)=\mathbf{e}\left(\lambda^{\prime}\right)^{*} \mathrm{e}(\lambda)$ and the atomic polarizibility tensor $\mathbf{X}(\omega)$ were defined above. In each of these terms we retain only those terms for which the exponential time variation vanishes.

\section{\$4.4 Nonadiabatic Corrections}

As discussed above, the effects of broadband radiation can be taken into account by replacing the field responsible for an induced moment by a filtered field $\mathscr{F}_{\lambda}(t)$. With this correction the formula for the Raman Hamiltonian becomes

$$
\begin{aligned}
W_{p^{\prime} p}^{r a m}= & -\frac{1}{4 \hbar} \sum_{\lambda \lambda^{\prime}} \alpha_{p^{\prime} p}\left(\lambda^{\prime}, \lambda\right) \mathscr{E}_{\lambda^{\prime}} \mathscr{F}_{\lambda} \\
& \times \exp \left[i\left(W_{p^{\prime}}-W_{p}+\omega_{\lambda^{\prime}}-\omega_{\lambda}\right) t\right] \exp \left[-i\left(K_{p^{\prime}}-K_{p}+k_{\lambda^{\prime}}-k_{\lambda}\right) z\right] .
\end{aligned}
$$

Because the polarizability correction alters the induced dipole moment (in distinction to the fixed transition dipole moments) it affects only one of the two fields that appear in this formula. That is, the correction is associated with the single detuning denominator of the polarizability rather than with the two dipole transition moments.

\section{$\S 4.5$ Propagation: Linear and Nonlinear Parts}

Traditional nonlinear optics introduces a separation of linear and nonlinear polarization fields

$$
\mathscr{S}_{\lambda}=\operatorname{lin} \mathscr{P}_{\lambda}+n l \mathscr{P}_{\lambda}
$$

in which the linear term is responsible for dispersion (and absorption) and the nonlinear term is responsible for Raman and other frequency-changing processes. With the present approximations the linear part is, for a single species of nondegenerate atoms,

$$
\operatorname{lin} \mathscr{P}_{\lambda}=\sum_{p \lambda} \mathscr{V}_{\rho p} \rho_{p p}(\lambda, \lambda) \mathscr{F}_{\lambda}
$$

On using the definition of $\mathcal{F}_{\lambda}$ we obtain the expression

$$
\operatorname{lin} \mathcal{P}_{\lambda}(t)=\chi_{\lambda}\left(\omega_{\lambda}\right) \mathscr{F}_{\lambda}(t)=\sum_{k} \frac{1}{k !}\left(\frac{d}{d \omega_{\lambda}}\right)^{k} \chi_{\lambda}\left(\omega_{\lambda}\right)\left(i \frac{d}{d t}\right)^{k} \mathcal{E}_{\lambda}(t)
$$

where the linear susceptibility is 


$$
\chi_{\lambda}\left(\omega_{\lambda}\right)=\sum_{p} \mathcal{N} \rho_{p p} \alpha_{p p}(\lambda, \lambda)
$$

We thereby obtain the propagation equation

$$
\left[\frac{d}{d z}+\frac{1}{c} \frac{d}{d t}\right] \mathscr{E}_{\lambda}=\left[i \frac{2 \pi \omega_{\lambda}}{c} \chi_{\lambda}\left(\omega_{\lambda}\right)-\frac{1}{v_{\lambda}^{\prime}} \frac{d}{d t}+\ldots\right] \mathscr{E}_{\lambda}+i \frac{2 \pi \omega_{\lambda}}{c} \pi l \mathscr{P}_{\lambda} .
$$

Here the ellipsis ... denotes higher time derivatives and the incremental group velocity $v_{\lambda}^{\prime}$ is defined as

$$
\frac{1}{v_{\lambda}^{\prime}}=\frac{1}{v_{\lambda}}-\frac{1}{c}=\frac{1}{c}\left[4 \pi \chi_{\lambda}\left(\omega_{\lambda}\right)+2 \pi \omega_{\lambda} \frac{d}{d \omega_{\lambda}} \chi_{\lambda}\left(\omega_{\lambda}\right)\right]
$$

The left-hand side of the equation is just the free-space propagation operator. The righthand side contains all of the effects of matter, as expressed by linear and nonlinear polarization.

\section{\$4.6 The Reference Frame}

When treating propagation of a single pulse, in the absence of Raman or other nonlinear processes, it is customary to introduce a reference frame that moves with the group velocity. In such a frame the pulse will remain moderately stationary. However, when one treats multiple pulses, each with a different group velocity, it proves more convenient to introduce a single reference frame that moves with the vacuum speed of light, $c$. In such a frame the propagation equation becomes

$$
\frac{d}{d z} \mathscr{E}_{\lambda}=i \mathscr{K}_{\lambda} \mathscr{E}_{\lambda}+i \frac{2 \pi \omega_{\lambda}}{c} n l \mathscr{P}_{\lambda} \text {. }
$$

where the linear dispersion operator $\mathscr{K}_{\lambda}$ is

$$
\begin{aligned}
\mathscr{K}_{\lambda} \mathcal{F}_{\lambda} & =\frac{2 \pi \omega_{\lambda}}{c} \chi_{\lambda}\left(\omega_{\lambda}\right) \mathscr{F}_{\lambda}(t) \\
& =\left[\frac{2 \pi \omega_{\lambda}}{c} \chi_{\lambda}\left(\omega_{\lambda}\right)+\frac{i}{v_{\lambda}} \frac{d}{d t}+\ldots\right] \mathcal{E}_{\lambda} .
\end{aligned}
$$

The presence of the linear polarization field causes a pulse envelope to move (and distort) in this moving window. This distortion (e.g. conversion of phase to amplitude modulation) is represented by the field $\mathscr{F}_{\lambda}(t)$ or the operator $\mathscr{K}_{\lambda}$. In particular, it is to be expected that the pump and Stokes envelopes will move at different speeds in any window. It is the nonlinear term that causes the Stokes field to grow. 


\section{\$5 Application to Three-Level Atoms}

We next consider application of the preceding formalism to an ensemble of randomly inoving three-level atoms. The third level is virtual, and does not appear explicitly in the equations of motion. Its effects enter through the polarizability. We exhibit the relevent Bloch equations, involving Raman coherences and Raman inversion, and incorporate these into the propagation equations by means of integrals. The Doppler broadening (and any homogeneous relaxation) enters the theory as a memory function, relating the present induced dipole moment to the past pulse history.

\section{\$5.1 The Stokes Source}

We illustrate these results by considering a simple three-state system, the lambda linkage

$$
1 \longmapsto 3 \longleftrightarrow 2
$$

in which the pump field $\mathscr{E}_{P}$ drives a transition between states 1 and 3 , while the Stokes wave $\mathscr{E}_{S}$ couples states 3 and 2 . State 1 is the initially populated state (typically the ground state), state 2 is a metastable state (unconnected with the ground state by direct dipole interaction), and state 3 becomes a virtual itate when the pump is sufficiently far from resonance. We define the pump and Stokes carrier frequencies $\omega_{P}$ and $\omega_{S}$ to obey the constraint

$$
E_{1}+\hbar \omega_{P}=E_{2}+\hbar \omega_{S}
$$

The differerce between pump and Stokes frequencies, the Raman frequency $\omega_{R}$, is the Bohr frequency for the (two-photon) $1 \longleftrightarrow 2$ transition:

$$
\hbar \omega_{R}=E_{2}-E_{1} .
$$

When the Stokes field is resonant with the 2,3 transition, we require the polarization envelopes

$$
\operatorname{res} \mathscr{P}_{S}=2 \mathscr{N}^{\operatorname{res}} \rho_{32} \mathrm{e}(S)^{*} \cdot\langle 2|\mathrm{~d}| 3\rangle \equiv 2 \mathscr{N} \text { res } \rho_{32} d_{23}
$$

When the field is not resonant with this transiticn we can eliminate explicit reference to state 3 by introducing appropriate polarizabilities. Those contributions constitute $n r \mathscr{P}_{\lambda}$, and they involve polarizabilities. The source terms for pump and Stokes are, for nondegenerate levels

$$
\operatorname{nr} \mathscr{P}_{P}=\sum_{P^{\prime}} \mathscr{N}\left[\rho_{11} \alpha_{11}\left(P, P^{\prime}\right)+\rho_{22} \alpha_{22}\left(P, P^{\prime}\right)\right] \mathscr{F}_{P^{\prime}}+\sum_{S^{\prime}} \mathscr{N} \rho_{21} \alpha_{12}\left(P, S^{\prime}\right) \mathscr{F}_{S^{\prime}}
$$

$$
{ }^{n r} \mathscr{P}_{S}=\sum_{S^{\prime}} \mathcal{N}^{\prime}\left[\rho_{11} \alpha_{11}\left(S, S^{\prime}\right)+\rho_{22} \alpha_{22}\left(S, S^{\prime}\right)\right] \mathscr{F}_{S^{\prime}}+\sum_{P^{\prime}} \mathcal{N}_{\rho_{12}} \alpha_{21}\left(S, P^{\prime}\right) \mathscr{F}_{P^{\prime}}
$$


These expressions exhibit two distinct contributions. The first sum, involving populations $\rho_{p p}$, contains the linear response of the medium, typically parameterized by an index of refraction and a group velocity. The second sum, involving the two-photon Raman coherence $\rho_{12}$, is the source of stimulated Raman scattering.

\section{$\$ 5.2$ The Anti-Stokes Source}

By creating an induced dipole moment at the Raman frequency $\omega_{R}$ the pump field not only produces Stokes radiation, at frequency $\omega_{S}=\omega_{P}-\omega_{R}$, it also produces anti-Stokes radiation at frequency

$$
\omega_{A}=\omega_{P}+\omega_{R} .
$$

The source term for this radiation is

$$
\begin{aligned}
n r \mathscr{P}_{A} & =\sum_{A^{\prime}} \mathcal{N}\left[\rho_{11} \alpha_{11}\left(A, A^{\prime}\right)+\rho_{22} \alpha_{22}\left(A, A^{\prime}\right)\right] \mathscr{F}_{A^{\prime}} \\
& +\sum_{P^{\prime}} \mathcal{N} \rho_{21} \alpha_{12}\left(A, P^{\prime}\right) \mathscr{F}_{P^{\prime}} \exp [i \Delta k z]
\end{aligned}
$$

where

$$
\Delta k=2 k_{P}-k_{A}-k_{S}
$$

is the phase mismatch between two pump photons and the Stokes-antiStokes pair.

\section{$\S 5.3$ The Pump Source}

When the pump is resonant with the 1,3 transition, we require the polarization envelopes

$$
\operatorname{res}_{\mathscr{P}_{P}}=2 \mathcal{N}^{\text {res }} \rho_{31} \mathrm{e}(P)^{*} \cdot\langle 1|\mathrm{~d}| 3\rangle \equiv 2 \mathcal{N}^{\text {res }} \rho_{31} d_{13}
$$

When the field is not resonant with this transition we can eliminate explicit reference to state 3 by introducing appropriate polarizabilities. Those contributions constitute ${ }^{n r} \mathscr{P}_{\lambda}$, and they involve polarizabilities. Both the Stokes and the anti-Stokes fields couple to the pump field, leading to the source (for nondegenerate levels)

$$
{ }^{n r} \mathscr{P}_{P}=\sum_{P^{\prime}} \mathcal{N}\left[\rho_{11} \alpha_{11}\left(P, P^{\prime}\right)+\rho_{22} \alpha_{22}\left(P, P^{\prime}\right)\right] \mathcal{F}_{P^{\prime}}
$$




$$
\begin{aligned}
& +\sum_{S^{\prime}} \mathcal{N}_{\rho_{21}} \alpha_{12}\left(P, S^{\prime}\right) \mathscr{F}_{S^{\prime}} \\
& +\sum_{A^{\prime}} \mathcal{N} \rho_{12} \alpha_{21}\left(P, A^{\prime}\right) \mathscr{F}_{A^{\prime}} \exp [-i \Delta k z]
\end{aligned}
$$

The anti-Stokes polarizability $\alpha_{12}(A, P)$ that occurs here differs from that for the Stokes radiation, $\alpha_{21}(S, P)$. When the pump is near resonant for the transition from ground level to excited (virtual) level, then the Stokes polarizability is near resonant, whereas the antiStokes polarizability is off resonance by the Raman frequency. Although many situations occur, particularly with Raman transitions in molecules, for which the Raman frequency is small and both Stokes and anti-Stokes radiation contribute, we here consider only the Stokes radiation.

\section{§5.4 Doppler and Sublevel Averages}

The vapor through which radiation passes typically contains several species of atoms (and isotopes), each of which contributes to the polarization field. Even a single species will have thermal motions and random orientations, and so will exhibit inhomogeneous relaxation of induced moments. We treat the motion by introducing a summation over Doppler shifts, and we treat the orientation degeneracy by averaging over magnetic quantum numbers.

To evaluate the effect of Doppler relaxation we assume that populations are independent of Doppler shift. This assumption is consistent with our neglect of population changes. With the inclusion of a Doppler distribution of atomic velocity components for coherences (but not for populations $\rho_{11}$ and $\rho_{22}$ ) we can write the Stokes source, for resonant and nonresonant atoms, as

$$
\begin{aligned}
\mathscr{P}_{S}(z, t) & =2 \text { res. } r d_{23} \int_{0}^{\infty} d \delta \mathscr{I}_{32}(\delta){ }^{r e s} \rho_{32}(z, t, \delta) \mathcal{E}_{S}(z, t) \\
& +\sum_{S^{\prime}}\left[n r \mathcal{r}_{1} \alpha_{11}\left(S, S^{\prime}\right)+n r \mathcal{r}_{2} \alpha_{22}\left(S, S^{\prime}\right)\right] \mathscr{F}_{S^{\prime}}(z, t) \\
& +n r \cdot r \sum_{P} \int_{-\infty}^{\infty} d \delta g_{12}(\delta){ }^{n r} \rho_{12}(z, t, \delta) \alpha_{21}(S, P) \mathscr{T}_{P}(z, t)
\end{aligned}
$$

where $g_{32}(\delta)$ is the one-photon Doppler profile and $g_{12}(\delta)$ is the two-photon (Raman) Doppler profile, for Doppler shift $\delta$. These functions have the normalization 


$$
\int d \delta g(\delta)=1
$$

It is customary to take the Doppler profile to be a Gaussian function of detuning, but this is not a neccessary form. In all cases the peak value $\pi g(0)$ is, by definition, the coherence time for Doppler relaxation.

To evaluate the effect of magnetic sublevels we must sum over degenerate final states and average over degenerate initial states. If we indicate the needed sum and average by the symbol $\{\ldots\}$ then the nonresonant contribution takes the form

$$
\begin{aligned}
& n r \mathscr{P}_{S}(z, t)=\sum_{S^{\prime}}\left[{ }^{n r} \mathcal{N}_{1}\left\{\alpha_{11}\left(S, S^{\prime}\right)\right\}+{ }^{n r} \mathcal{N}_{2}\left\{\alpha_{22}\left(S, S^{\prime}\right)\right\}\right] \mathscr{E}_{S^{\prime}}(z, t) \\
& +n r \vee \sum_{P} \int_{-\infty}^{\infty} d \delta g_{12}(\delta)\left\{{ }^{n r} \rho_{12}(z, t, \delta) \alpha_{21}(S, P)\right\} \mathcal{E}_{P}(z, t) .
\end{aligned}
$$

To carry out these sums we must express the various tensor matrix elements of the atom and the polarization vectors of the field in an angular momentum basis, use the WignerEckart theorem to extract the dependence on magnetic sublevels and polarization direction, and carry out the surns.

\section{§5.5 The Nonresonant (Raman) Bloch Equations}

Consider the nonresonant (Raman) equations, in which level 3 has been adiabatically eliminated to produce a Raman Hamiltonian. The atomic variables are the populations $\rho_{11}$ and $\rho_{22}$ and the complex-valued coherence $\rho_{12}$. The equations for populations are:

$$
\begin{aligned}
& \frac{d}{d \mathrm{t}} \rho_{22}=-\Gamma_{22} \rho_{22}-i\left[\mathcal{Q}_{21} \rho_{12}-\mathscr{Q} \mathcal{L}_{12} \rho_{21}\right]+h_{22} \\
& \frac{d}{d^{\prime}} \rho_{11}=-\Gamma_{11} \rho_{11}-i\left[\mathscr{W}_{12} \rho_{21}-\mathscr{W}{ }_{21} \rho_{12}\right]+h_{11} .
\end{aligned}
$$

The equation for the Raman coherence is

$$
\left.\frac{d}{d t} \rho_{12}=-\left[i \delta_{12}+\Gamma_{12}\right] \rho_{12}-i \mathscr{W}\right)_{12}\left[\rho_{22}-\rho_{11}\right]+h_{12} .
$$

Here the matrix $W$ is the Raman Hamiltonian and the quantities $h_{i j}$ are Langevin noise sources associated with the homogeneous relaxation terms $\Gamma_{i j}$. (Such noise terms are needed whenever we replace quantum mechanical operator equations with expectation values, as is done in presenting equations of motion for a density matrix.) With the inclusion of both Stokes and anti-Stokes fields (but not higher order fields, such as second Stokes) the element of this Hamiltonian that produces transitions is, in the polarizability approximation, 


$$
\begin{gathered}
W_{21}=-\frac{1}{4 \hbar}\left\langle 2\left|\mathbf{X}\left(\omega_{P}\right)\right| 1\right\rangle: \in(S, P) \mathscr{E}_{S}^{*} \mathscr{E}_{P} \exp \left[-i\left(K_{2}-K_{1}+k_{S}-k_{P}\right) z\right] \\
-\frac{1}{4 \hbar}\left\langle 2\left|\mathbf{X}\left(\omega_{A}\right)\right| 1\right\rangle: \in(P, A) \mathcal{E}_{P}^{*} \mathscr{E}_{A} \quad \exp \left[-i\left(K_{2}-K_{1}+k_{P}-k_{A}\right) z\right]
\end{gathered}
$$

In general the matrix element $W_{21}$ recieves contributions frcm all pairs of fields whose frequencies combine to produce the Raman frequency $\omega_{R}$. Here we consider only Stokes, anti-Stokus, and pump fields. Note that, when the pump radiation is near resonant, the matrix elements of $\mathbf{X}\left(\omega_{P}\right)$ are larger than those of $\mathbf{X}\left(\omega_{A}\right)$, roughly by the ratio $\omega_{R} / \Delta$,

$$
\begin{aligned}
& \left\langle 2\left|\mathbf{X}\left(\omega_{P}\right)\right| 1\right\rangle \simeq \frac{\langle 2|\mathrm{~d}| 3\rangle\langle 3|\mathrm{~d}| 1\rangle}{E_{3}-E_{1}-\omega_{P}} \equiv \frac{\langle 2|\mathrm{~d}| 3\rangle\langle 3|\mathrm{~d}| 1\rangle}{\Delta} \\
& \left\langle 1\left|\mathbf{X}\left(\omega_{S}\right)\right| 2\right\rangle \simeq \frac{\langle 1|\mathrm{~d}| 3\rangle\langle 3|\mathrm{~d}| 2\rangle}{E_{3}-E_{2}-\omega_{S}} \equiv \frac{\langle 1|\mathrm{~d}| 3\rangle\langle 3|\mathrm{~d}| 2\rangle}{\Delta} \\
& \left\langle 2\left|\mathbf{X}\left(\omega_{A}\right)\right| 1\right\rangle \simeq \frac{\langle 2|\mathrm{~d}| 3\rangle\langle 3|\mathrm{~d}| 1\rangle}{E_{3}-E_{1}-\omega_{A}} \equiv \frac{\langle 2|\mathrm{~d}| 3\rangle\langle 3|\mathrm{~d}| 1\rangle}{\Delta+\omega_{R}}
\end{aligned}
$$

and so under these conditions the Anti-Stokes contribution is small. Note also the possibility of destructive interference between the two combinations, $\mathscr{G}_{S}{ }^{*} \mathscr{S}_{P}$ and $\mathscr{S}_{P}{ }^{*} \mathscr{S}_{A}$, that drive the Raman coherence $\rho_{12}$. detuning:

The detuning $\delta_{12}$ that occurs with the Raman coherence is the two-photon (Raman)

$$
\delta_{12}=-\omega_{21}+\omega_{P}-\omega_{S}+W_{11}-W_{22}
$$

This detuning (a dynamic Stark shift) depends on $W_{11}$ and $W_{22}$ : it is detuning relative to dynamically shifted energy levels. This shift receives contributions from all fields, but in the model considered here (weak Stokes) it is the pump pulse that is responsible for the shift:

$$
W_{n n}=-\frac{1}{4 \hbar}\left\langle n\left|\mathbf{X}\left(\omega_{P}\right)\right| n\right\rangle: \in(P, P)\left|\mathscr{E}_{P}\right|^{2}
$$

When we consider pump, Stokes and anti-Stokes waves within the usual polarizability approximation the equation for the Raman coherence $\rho_{12} \equiv \rho_{12}(z, t, \delta)$ reads

$$
\begin{aligned}
& \frac{d}{d t} \rho_{12}=-\left[i \delta-i \Delta_{\text {stark }}+\Gamma_{12}\right] \rho_{12}+h_{12}+\frac{i}{4 \hbar}\left[\rho_{22}-\rho_{11}\right] \sum_{S P} \alpha_{21}(S, P)^{*} \mathcal{S}_{P}^{*} \mathcal{E}_{S} \\
& +\frac{i}{4 \hbar}\left[\rho_{22}-\rho_{11}\right] \sum_{P A} \alpha_{21}(P, A)^{*} \mathcal{E}_{A}^{*} \mathcal{E}_{P} \exp \left(i \Delta k_{P A} z\right)
\end{aligned}
$$


where the two-photon Doppler shift is

$$
\hbar \delta=E_{2}-E_{1}-\hbar \omega_{P}+\hbar \omega_{S}
$$

The equation for population in the (metastable) excited state is

$$
\begin{aligned}
\frac{d}{d t} \rho_{22}= & -\Gamma_{22} \rho_{22}-\sum_{S P} 2 \mathscr{G} m\left[\rho_{12} \alpha_{21}(S, P) \mathcal{E}_{S}^{*} \mathcal{E}_{P}\right] \\
& -\sum_{A P} 2 \mathscr{J} m\left[\rho_{12} \alpha_{21}(A, P) \mathcal{E}_{A}{ }^{*} \mathcal{E}_{P} \exp \left(-i \Delta k_{P A} z\right)\right] .
\end{aligned}
$$

Our procedure is:

1. Obtain an integral expression for the Raman coherence $\left(\rho_{12}\right)$.

2. Assume the populations $\left(\rho_{11}, \rho_{22}\right)$ are fixed at initial values.

The fixed-population approximation is acceptable as long as changes to a given atom remain small during exposure to a pulse.

\section{\$5.6 The Macroscopic Raman Coherences and Inversion}

The equation for the nonlinear (and nonresonant) contribution to the Stokes polcrization envelope in the presence of Doppler broadening can be written as

$$
n l \mathscr{P}_{S}(z, t)=n r \mathcal{N} \sum_{P} \alpha_{21}(S, P) Q(z, t) \mathscr{F}_{P}(z, t)
$$

where $Q(z, t)$ is the macroscopic Raman coherence, an average over single-atom coherences $Q(z, t, \delta)$ for those atoms whose two-photon Doppler shifts are $\delta:$

$$
Q(z, t) \equiv \int_{-\infty}^{\infty} d \delta g_{12}(\delta) Q(z, t, \delta), \quad Q(z, t, j)={ }^{n r} \rho_{12}(z, t, \delta) .
$$

The Bloch equation provides, for specified fields, coupled equations for the single atom coherences and the single atom populations. Rather than solve these coupled equations, we introduce an approximation for the populations: we neglect dependence of populations upon detuning. This approximation amounts to assuming that the Doppler broadening acts as homogeneous relaxation, rather than as inhomogeneous relaxation. We define the macroscopic Raman inversion $w(z, t)$ as

$$
w(z, t)=\rho_{22}(z, t)-\rho_{11}(z, t) .
$$


Within this approximation the equation for the single-atom Raman coherence, in the absence of anti-Stokes waves, is

$$
\begin{gathered}
\frac{\partial}{\partial t} Q(z, t, \delta)=-\left[i \delta-i \Delta_{s t a r k}+\Gamma_{12}\right] Q(z, t, \delta)+h_{12}(t) \\
+\frac{i}{4 \hbar} w(z, t) \sum_{S P} \alpha_{21}(S, P)^{*} \mathscr{F}_{P}(z, t)^{*} \mathscr{E}_{S}(z, t) .
\end{gathered}
$$

As above, $\delta$ is the two-photon Raman detuning. The equation for Raman inversion we take to be the equation for the Doppler averaged inversion in the absence of spontaneous emission:

$$
\left.\frac{\partial}{\partial t} w(z, t)=-\sum_{S P} 2 \mathscr{I}_{m[} \alpha_{21}(S, P) \mathscr{E}_{S}(z, t)^{*} \mathscr{F}_{P}(z, t) Q(z, t)\right] .
$$

\section{$\S 5.7$ Integral Equation}

With the phase choice $K_{2}-K_{1}=k_{P}-k_{S}$ the integral equation for the single-atom Raman coherence at given time $t$ and given detuning is

$$
\begin{aligned}
& Q(t, \delta)={ }^{n o i s e} Q(t, \delta) \\
& +\frac{i}{4 \hbar} \sum_{P S} \alpha_{12}(P, S) \int_{t_{0}}^{t} d t^{\prime} \exp \left[-\left(i \delta+\Gamma_{12}\right)\left(t-t^{\prime}\right)-i \phi_{12}(t)+i \phi_{12}\left(t^{\prime}\right)\right] w\left(t^{\prime}\right) \mathscr{F}_{P}\left(t^{\prime}\right)^{*} \mathscr{E}_{S}\left(t^{\prime}\right) .
\end{aligned}
$$

Here $\delta$ denotes the two-photon detuning in the absence of the dynamic Stark shift, and $\phi_{12}(t)$ is the phase shift produced by that shift:

$$
\phi_{12}(t)=\int_{t_{0}}^{t} d t^{\prime} \Delta_{S t a r k}\left(t^{\prime}\right)=-\frac{1}{4 \hbar}\left[\alpha_{22}(P, P)-\alpha_{11}(P, P)\right] \int_{t_{0}}^{t} d t^{\prime}\left|\mathcal{E}_{P}\left(t^{\prime}\right)\right|^{2}
$$

The time $t_{0}$ is the initialization time (when the initial conditions apply, before the radiation arrives.) We shall take the limit $t_{0} \rightarrow-\infty$. The first term,

$$
\begin{aligned}
& { }^{n o i s e} Q(t, 0)=Q\left(t_{0}, \delta\right) \exp \left[-\left(i \delta+\Gamma_{12}\right)\left(t-t_{0}\right)-i \phi_{12}(t)\right] \\
& +\int_{t_{0}}^{t} d t^{\prime} \exp \left[-\left(i \delta+\Gamma_{12}\right)\left(t-t^{\prime}\right)-i \phi_{12}(t)+i \phi_{12}\left(t^{\prime}\right)\right] h_{12}\left(t^{\prime}\right),
\end{aligned}
$$

involving $Q\left(z, t_{0}\right)$ and $h_{12}\left(t^{\prime}\right)$, introduces the fluctuations that are responsible for the gener- 
ation of Stokes radiation from spontaneous emission, i.e. noise. 


\section{$\S 5.8$ The Memory Function}

When the populations changes can be regarded as independent of detuning (i.e. the population relaxation behaves as homogeneous relaxation) the only dependence on detuning occurs explicitly in the exponentiated phase. Under this condition we can solve the Bloch equation for each detuning and integrate over detunings. To simplify the results we here neglect the Stark shifts. Then the entire influence of relaxation appears in an integral, over detuning $\delta$, of the Doppler profile combined with the homogeneous relaxation rate:

$$
\begin{aligned}
\mathscr{G}(\tau)= & \int d \delta g(\delta) \exp \left[-\left(i \delta+\Gamma_{12}\right) \tau\right] \text { for } \tau>0 \\
& =1 \text { for } \tau=1 \\
& =0 \text { for } \tau<0
\end{aligned}
$$

For subsequent convenience we define this function as vanishing for negative values of the argument. This function is normalized to have unit peak value, $\mathscr{G}(0)=1$, and so the integral of $\mathscr{G}(\tau)$ provides a measure of the Raman coherence time,

$$
\tau_{R}=\int_{-\infty}^{\infty} d \tau \mathscr{G}(\tau)=\pi g(0)
$$

We refer to $\mathscr{G}(\tau)$ as the memory function. Upon extending the initial time $t_{0}$ to the infinite past we can express the result of this integration as

$$
\begin{aligned}
& Q(z, t)={ }^{\text {noise }} Q(z, t) \\
& +\frac{i}{4 \hbar} \sum_{P S} \alpha_{12}(P, S) \int_{-\infty}^{\infty} d t^{\prime} \mathscr{G}_{12}\left(t-t^{\prime}\right) w\left(z, t^{\prime}\right) \mathscr{F}_{P}\left(z, t^{\prime}\right)^{*} \mathcal{E}_{S}\left(z, t^{\prime}\right) .
\end{aligned}
$$

where the noise contribution to the macroscopic Raman coherence is

$$
\text { noise } Q(z, t)=\mathscr{G}_{12}\left(t-t_{0}\right) Q\left(z, t_{0}\right)+\int_{-\infty}^{\infty} d t^{\prime} \mathscr{G}\left(t-t^{\prime}\right) h_{12}\left(t^{\prime}\right)
$$

The Stokes polarization field that results from this expression is 


$$
\begin{aligned}
& n l \mathscr{P}_{S}(z, t)= n r \mathcal{N} \sum_{P} \alpha_{21}(S, P) \mathscr{F}_{P}(z, t) \mathscr{G}_{12}\left(t-t_{0}\right) Q\left(z, t_{0}\right) \\
&+n r \mathcal{N} \sum_{P} \alpha_{21}\left(S, i^{\prime}\right) \mathscr{F}_{\rho}(z, t) \int_{-\infty}^{\infty} d t^{\prime} \mathscr{G}\left(t-t^{\prime}\right) h_{12}\left(t^{\prime}\right) \\
&+\frac{i}{4 \hbar} \mathcal{N} \sum_{P P^{\prime} S^{\prime}} \alpha_{21}(S, P) \alpha_{12}\left(P^{\prime} S^{\prime} ;\right. \\
& \times \mathscr{F}_{P}(z, t) \int_{-\infty}^{\infty} d t^{\prime} \mathscr{G}\left(t-t^{\prime}\right) w\left(z, t^{\prime}\right) \mathscr{F}_{P^{\prime}}\left(\cdot, t^{\prime}\right)^{*} \mathscr{E}_{S^{\prime}}\left(z, t^{\prime}\right) .
\end{aligned}
$$

The first term describes the initial polarization at position $z$ (a random variable). The second term describes the effects of the Langevin noise source that must be present when there is homogeneous damping of the atomic equations. The final term is the SRS source. As can be seen, the polarization envelope receives contributions from all pump and Stokes polarizations, as indicated by the sum on $P P^{\prime} S^{\prime}$. 


\section{$\S 6$ The Propagation Equations}

We here present the coupled Maxwell-Bloch equations appropriate to Raman processes for an ensemble of two-level atoms. We show the correction that is needed to correct the polarizability for pump bandwidth effects. We comment on averages over magnetic sublevels, and on the special case of short relaxation time, leading to the definition of steady-state gain coefficient.

\section{$\S 6.1$ The Maxwell-Bloch Equations}

Within the polarizability approximation (valid for pulses whose frequency components do not overlap resonances), the envelope propagation equations introduce atomic effects by means of diagonal and off-diagonal elements of two matrices: the density matrix (expressing atomic excitation and coherence in the presence of fields), and the polarizability matrix (expressing various induced moments). Pump propagation involves only the diagonal elements of these matrices (populations and traditional polarizabilities). Stokes generation via Raman processes involves the off-diagonal elements of these two matrices. Specifically, for Stokes generation induced by pump field in a three-state atom, we require the combination $\rho_{12} \alpha_{21}\left(\omega_{P}\right)$ associated with the atomic transition $1 \rightarrow 2$ at frequency $\omega_{R}=$ $\omega_{P}-\omega_{S}$.

The pump field that produces Stokes radiation will also produce Anti-Stokes radiation, at frequency $\omega_{A}=\omega_{P}+\omega_{R}$. The source term for this process involves the product $\rho_{21}$ $\alpha_{12}\left(\omega_{P}\right)$. Once the Anti-Stokes field is present, it can act, by means of the pump-induced dipole moment, as an additional source for the Stokes field. The growth of the Stokes field produced by an Anti-Stokes field involves the combination $\rho_{21} \alpha_{12}\left(\omega_{A}\right)$

The propagation equations that must be treated, for an ensemble of nondegenerate two-state Doppler-broadened atoms, are (with the neglect of anti-Stokes coupling) the two field equations (versions of Maxwell's equations in the presence of matter)

$$
\begin{aligned}
\frac{\partial}{\partial z} \mathcal{E}_{S}(z, t) & =+i \frac{2 \pi \omega_{S}}{c} \sum_{S^{\prime}}\left[\mathcal{N}_{1} \alpha_{11}\left(S, S^{\prime}\right)+\mathcal{V}_{2} \alpha_{22}\left(S, S^{\prime}\right)\right] \mathscr{F}_{S^{\prime}}(z, t) \\
& +i \frac{2 \pi \omega_{S}}{c} \sum_{P} \mathcal{N} \alpha_{21}(S, P) \mathscr{F}_{P}(z, t) Q(z, t) \\
\frac{\partial}{\partial z} \mathcal{E}_{P}(z, t) & =+i \frac{2 \pi \omega_{P}}{c} \sum_{P^{\prime}}\left[\mathscr{N}_{1} \alpha_{11}\left(P, P^{\prime}\right)+\mathcal{F}_{2} \alpha_{22}\left(P, P^{\prime}\right)\right] \mathscr{F}_{P^{\prime}}(z, t) \\
& +i \frac{2 \pi \omega_{P}}{c} \sum_{S} \mathcal{N}_{\alpha_{12}(P, S) \mathscr{F}_{S}(z, t) Q(z, t)^{*}}
\end{aligned}
$$


where the atomic density $\mathcal{N}=\mathcal{N}_{1}+\mathcal{N}_{2}$ is that of nonresonant atoms. The macroscopic Raman coherence is expressible as a macroscopically averaged integral solution of singleatom Bloch equations

$$
\begin{aligned}
& Q(z, t)=\text { noise } Q(z, t) \\
& +\frac{i}{4 \hbar} \sum_{P S} \alpha_{12}(P, S) \int_{-\infty}^{\infty} d t^{\prime} \mathscr{G}_{12}\left(t-t^{\prime}\right) w\left(z, t^{\prime}\right) \mathscr{F}_{P}\left(z, t^{\prime}\right)^{*} \mathcal{E}_{S}\left(z, t^{\prime}\right)
\end{aligned}
$$

where

$$
{ }^{n o i s e} Q(z, t)=\mathscr{G}_{12}\left(t-t_{0}\right) Q\left(z, t_{0}\right)+\int_{-\infty}^{\infty} d t^{\prime} \mathscr{G}\left(t-t^{\prime}\right) h_{12}\left(t^{\prime}\right)
$$

To this equation we add the Bloch equation for macroscopic Raman inversion,

$$
w(z, t)=w\left(z, t_{0}\right)-\sum_{S P} \int_{-\infty}^{t} d t^{\prime} \mathscr{g} m\left[\alpha_{21}(S, P) \mathscr{E}_{S}\left(z, t^{\prime}\right)^{*} \mathscr{F}_{P}\left(z, t^{\prime}\right) Q\left(z, t^{\prime}\right)\right]
$$

or, with the elimination of $Q(z, t)$,

$$
\begin{aligned}
& w(z, t)= \text { noise }_{w}(z, t) \\
&-\frac{1}{4 \hbar} \mathscr{R e} \sum_{S P S^{\prime} P^{\prime}} \alpha_{12}(P, S) \alpha_{21}\left(S^{\prime}, P^{\prime}\right) \int_{-\infty}^{t} d t^{\prime} \int_{-\infty}^{\infty} d t^{\prime \prime} \mathscr{G}_{12}\left(t^{\prime}-t^{\prime \prime}\right) \\
& \quad \times \mathcal{E}_{S^{\prime}}\left(z, t^{\prime}\right)^{*} \mathcal{E}_{S}\left(z, t^{\prime \prime}\right) \mathscr{F}_{P^{\prime}}\left(z, t^{\prime}\right) \mathscr{F}_{P}\left(z, t^{\prime \prime}\right)^{*} w\left(z, t^{\prime \prime}\right)
\end{aligned}
$$

As discussed above, the fields $\mathscr{F}_{\lambda}$ are frequency-filtered versions of the field $\mathscr{E}_{\lambda}$, introduced to account for finite bandwidth effects:

$$
\mathscr{F}_{\lambda}(t)=\int d \omega_{1} \frac{\exp \left[-i \omega_{1} t\right]}{1-\left(\omega_{1} / \Delta_{31 \lambda}\right)} \mathscr{C}_{\lambda}\left(\omega_{1}\right)
$$




\section{$\S 6.2$ Isotropic Medium}

We consider an isotropic medium. For such a system the orientation average of the polarizability $\alpha_{p p}\left(\lambda, \lambda^{\prime}\right)$ vanishes unless the polarizations of $\lambda$ and $\lambda^{\prime}$ are the same. Upon using this property and substituting the definition of $Q$ we obtain the equations

$$
\begin{aligned}
& \frac{\partial}{\partial z} \mathscr{E}_{S}(z, t)=+i \mathscr{K}_{S} \mathscr{S}_{S}(z, t)+\text { noise } \\
& \quad-\sum_{P P^{\prime} S^{\prime}} \kappa_{S}\left(S, P ; S^{\prime}, P^{\prime}\right) \int_{-\infty}^{\infty} d t^{\prime} \mathscr{G}\left(t-t^{\prime}\right) w\left(z, t^{\prime}\right) \mathscr{F}_{P^{\prime}}\left(z, t^{\prime}\right)^{*} \mathscr{E}_{S^{\prime}}\left(z, t^{\prime}\right) \mathscr{F}_{P}(z, t) \\
& \frac{\partial}{\partial z} \mathscr{E}_{P}(z, t)=+i \mathscr{K}_{P} \mathscr{E}_{P}(z, t) \\
& \quad+\sum_{P^{\prime} S^{\prime} S} \kappa_{P}\left(P, S ; P^{\prime}, S^{\prime}\right) \int_{-\infty}^{\infty} d t^{\prime} \mathscr{G}\left(t-t^{\prime}\right) w\left(z, t^{\prime}\right) \mathscr{F}_{P}\left(z, t^{\prime}\right) \mathscr{E}_{S^{\prime}}\left(z, t^{\prime}\right)^{*} \mathscr{F}_{S}(z, t)
\end{aligned}
$$

where the (linear) dispersion operator $\mathscr{K}_{\lambda}$ is defined by the equation

$$
\begin{aligned}
\mathscr{R}_{\lambda} \mathcal{E}_{\lambda} & =\frac{2 \pi \omega_{\lambda}}{c} \chi_{\lambda}\left(\omega_{\lambda}\right) \mathscr{F}_{\lambda}(t) \\
& =\frac{2 \pi \omega_{\lambda}}{c}\left[\mathcal{N}_{1} \alpha_{11}(\lambda, \lambda)+\mathcal{N}_{2} \alpha_{22}(\lambda, \lambda)\right] \mathscr{F}_{\lambda}(t) \\
\mathscr{F}_{\lambda}(t) & =\left[1+\frac{i}{\Delta} \frac{\partial}{\partial t}+\ldots\right] \mathcal{E}_{\lambda}(t)
\end{aligned}
$$

and the Raman coupling coefficients are

$$
\begin{aligned}
& \kappa_{S}\left(S, P ; S^{\prime}, P^{\prime}\right)=\mathcal{N} \frac{\pi \omega_{S}}{2 \hbar c} \alpha_{12}(P, S)^{*} \alpha_{12}\left(P^{\prime}, S^{\prime}\right) . \\
& \kappa_{P}\left(P, S ; P^{\prime}, S^{\prime}\right) \equiv \mathcal{N} \frac{\pi \omega_{P}}{2 \hbar c} \alpha_{12}(P, S) \alpha_{12}\left(P^{\prime}, S^{\prime}\right)^{*}=\frac{\omega_{P}}{\omega_{S}} \kappa_{S}\left(S, P ; S^{\prime} P^{\prime}\right)^{*} .
\end{aligned}
$$

The first term on the right hand side of each propagation equation is the dispersion (and absorption), including effects of the group velocity and of pulse distortion. The second term of the Stokes equation is the noise source. With the neglect of homogeneous relaxation this term is

$$
\text { noise }_{S}=i \frac{2 \pi \omega_{S}}{c} \sum_{P} \mathcal{N} \alpha_{21}(S, P) \mathscr{F}_{P}(z, t) \mathscr{G}_{12}\left(t-t_{0}\right) Q\left(z, t_{0}\right)
$$


The final term of each equation is the stimulated Raman effect: Stokes gain together with pump depletion. By combining the noise source with the SRS term we obtain the equation

$$
\begin{aligned}
& \frac{\partial}{\partial z} \mathscr{E}_{S}(z, t)=+i \mathscr{K}_{S} \mathcal{E}_{S}(z, t)+\text { noise } \\
& \quad-\sum_{P P^{\prime} S^{\prime}} \kappa_{S}\left(S, P ; S^{\prime}, P^{\prime}\right) \mathscr{F}_{P}(z, t) \int_{-\infty}^{\infty} d t^{\prime} \mathscr{G}\left(t-t^{\prime}\right) w\left(z, t^{\prime}\right) \mathscr{F}_{P^{\prime}}\left(z, t^{\prime}\right)^{*} \mathscr{E}_{S^{\prime}}\left(z, t^{\prime}\right)
\end{aligned}
$$

The equation for population change is

$$
\begin{aligned}
w(z, t) & =\text { noise }_{w}(z, t)-\mathscr{R e} \sum_{S P S^{\prime} P^{\prime}} \frac{\kappa_{P}\left(P, S ; P^{\prime} S^{\prime}\right)}{2 c \mathcal{N} \pi \omega_{P}} \int_{-\infty}^{t} d t^{\prime} \int_{-\infty}^{\infty} d t^{\prime \prime} \mathscr{G}_{12}\left(t^{\prime}-t^{\prime \prime}\right) \\
& \times \mathscr{E}_{S^{\prime}}\left(z, t^{\prime}\right)^{*} \mathcal{E}_{S}\left(z, t^{\prime \prime}\right) \mathscr{F}_{P^{\prime}}\left(z, t^{\prime}\right) \mathscr{F}_{P}\left(z, t^{\prime \prime}\right)^{*} w\left(z, t^{\prime \prime}\right)
\end{aligned}
$$

\section{$\S 6.3$ Sublevel Averages}

When orientation degeneracy is present these equations must be averaged over magnetic sublevels. Because we have neglected any possible field induced level shift in the phase of the memory function and have neglected any change in populations with time, the time variation of the coherence and of the polarization envelope is the same for any orientation. We can therefore average the propagation equations over sublevels, as in the expressions

$$
\begin{aligned}
& \kappa_{P}\left(P, S ; P^{\prime}, S^{\prime}\right) \equiv \mathcal{N} \frac{\pi \omega_{P}}{2 \hbar c}\left\{\alpha_{12}(P, S) \alpha_{12}\left(P^{\prime}, S^{\prime}\right)^{*}\right\} \\
& \kappa_{S}\left(S, P ; S^{\prime}, P^{\prime}\right)=\mathcal{N} \frac{\pi \omega_{S}}{2 \hbar c}\left\{\alpha_{12}(P, S)^{*} \alpha_{12}\left(P^{\prime}, S^{\prime}\right)\right\}
\end{aligned}
$$

We earlier used the property of isotropic matter,

$$
\left\{\alpha_{p p}\left(\lambda, \lambda^{\prime}\right)\right\}=0 \text { if } \lambda^{\prime} \neq \lambda
$$

\section{§6.4 Steady State Limit}

Frequently Raman processes are considered for conditions such that the memory function selects only short times, and the memory integral can then be replaced by instantaneous values. Under these conditions, and with the neglect of the polarizability correction and the assumption of single pump and Stokes modes, one has the equations

$$
\begin{aligned}
& \frac{\partial}{\partial z} \mathscr{E}_{S}(z, t)=i \mathscr{K}_{S} \mathcal{E}_{S}(z, t)-w \kappa_{S} \tau_{R}\left|\mathscr{S}_{P}(z, t)\right|^{2} \mathcal{E}_{S}(z, t) \\
& \frac{\partial}{\partial z} \mathscr{E}_{P}(z, t)=i \mathscr{K}_{P} \mathscr{S}_{P}(z, t)+w \kappa_{P} \tau_{R}\left|\mathscr{S}_{S}(z, t)\right|^{2} \mathscr{S}_{P}(z, t)
\end{aligned}
$$


where $\tau_{R}$ is the Raman coherence time defined above [the time integral of the memory function $\mathscr{G}(\tau)^{\prime}$.

$$
\begin{aligned}
& \kappa_{S}=\mathcal{N} \frac{\pi \omega_{S}}{2 \hbar c}\left\{\left|\alpha_{12}(P, S)\right|^{2}\right\} \\
& \kappa_{P}=\mathcal{N} \frac{\pi \omega_{P}}{2 \hbar c}\left\{\left|\alpha_{12}(P, S)\right|^{2}\right\}=\frac{\omega_{P}}{\omega_{S}} \kappa_{S}
\end{aligned}
$$

are coupling coefficients and

$$
W \equiv\left[\rho_{22}-\rho_{11}\right]
$$

is the constant macroscopic Raman inversion. As above, $\mathcal{N}$ refers to nonresonant atoms. The traditional steady-state Stokes gain coefficient $g_{s s}$, defined such that for constant pump intensity

$$
\frac{\partial}{\partial z} \mathcal{E}_{S}=\frac{1}{2} g_{s s} \mathcal{E}_{S}
$$

is identifiable from these expressions:

$$
g_{s s}=2|\pi| \kappa_{S} \tau_{R}\left|\mathcal{E}_{P}\right|^{2}=\mathcal{N}|\pi| \frac{\pi \omega_{S}}{\hbar c}\left\{\left|\alpha_{12}(P, S)\right|^{2}\right\}\left|\mathcal{E}_{P}(z, t)\right|^{2} \tau_{R}
$$

These equations predict that, given sufficient distance, an incident pump pulse will convert (entirely) into Stokes radiation. 


\section{$\S 7$ Fluence}

Treatments of steady-state Raman processes, involving continuous illumination, typically present equations and results in terms of intensity or irradiance. When dealing with pulse excitation it of ten proves more useful to present the theory in terms of the time integral of pulse intensity, i.e. the pulse fluence. We here discuss the relevent equations.

\section{\$7.1 The Fluence Equations}

An important measure of Stokes growth, or of pump depletion, is the fluence contained in a pulse at any position. We define the envelope product

$$
\mathscr{R}_{\lambda \lambda^{\prime}}(z, t) \equiv \mathscr{E}_{\lambda}(z, t)^{*} \mathscr{S}_{\lambda^{\prime}}(z, t)
$$

and the (unnormalized) fluence

$$
F_{\lambda}(z)=\int_{-\infty}^{\infty} d t \mathscr{R}_{\lambda \lambda}(z, t)=\int_{-\infty}^{\infty} d t\left|\mathcal{E}_{\lambda}(z, t)\right|^{2}
$$

We then obtain the equations

$$
\begin{aligned}
\frac{d}{d z} F_{P}(z) & =-2 \mathscr{R} e \int_{-\infty}^{\infty} d t \mathcal{E}_{P}(z, t)^{*} \mathscr{K}_{P} \mathcal{E}_{P}(z, t) \\
& -2 \sim \mathcal{} \frac{2 \pi \omega_{P}}{c} g_{m} \sum_{S} \alpha_{12}(P, S) \int_{-\infty}^{\infty} d t Q(z, t)^{*} \mathscr{R}_{P S}(z, t) \\
\frac{d}{d z} F_{S}(z) & =-2 \mathscr{R}_{e} \int_{-\infty}^{\infty} d t \mathcal{E}_{S}(z, t)^{*} \mathscr{K}_{s} \mathcal{E}_{S}(z, t) \\
& -2 \mathcal{N} \frac{2 \pi \omega_{S}}{c} g_{m} \sum_{S} \alpha_{12}(P, S)^{*} \int_{-\infty}^{\infty} d t \mathscr{R}_{P S}(z, t)^{*} Q(z, t)
\end{aligned}
$$

The needed Raman coherence is

$$
Q(z, t)=\text { noise }+\frac{i}{4 \hbar} w(z, t) \sum_{P^{\prime} S^{\prime}} \alpha_{12}\left(P^{\prime}, S^{\prime}\right) \int_{-\infty}^{\infty} d t^{\prime} \mathscr{G}\left(t-t^{\prime}\right) \mathscr{R}_{P^{\prime} S^{\prime}}\left(z, t^{\prime}\right)
$$

Upon substituting the integral form for the coherence we can write the equations as 


$$
\begin{aligned}
& \frac{d}{d z} F_{S}(z)=-2 \mathscr{R e} \int_{-\infty}^{\infty} d t \mathscr{E}_{S}(z, t)^{*} \mathscr{K}_{s} \mathcal{E}_{S}(z, t) \\
& -\mathcal{N} w \frac{2 \pi \omega_{S}}{2 \hbar c} R_{e} \sum_{P^{\prime} S^{\prime} S} \alpha_{12}(P, S)^{*} \alpha_{12}\left(P^{\prime}, S^{\prime}\right) \\
& \times \int_{-\infty}^{\infty} d t \mathscr{R}_{S P}(z, t) \int_{-\infty}^{\infty} d t^{\prime} \mathscr{G}\left(t-t^{\prime}\right) \mathscr{R}_{P^{\prime} S^{\prime}}\left(z, t^{\prime}\right) \\
& \frac{d}{d z} F_{P}(z)=-2 \mathscr{R} e \int_{-\infty}^{\infty} d t \mathscr{E}_{P}(z, t)^{*} \mathscr{K}_{P} \mathcal{E}_{P}(z, t) \\
& +\mathcal{N}_{w} \frac{2 \pi \omega_{P}}{2 \hbar c} \mathscr{R e} \sum_{P^{\prime} S^{\prime} S} \alpha_{12}(P, S) \alpha_{12}\left(P^{\prime}, S^{\prime}\right)^{*} \\
& \times \int_{-\infty}^{\infty} d t \mathscr{R}_{P S}(z, t) \int_{-\infty}^{\infty} d t^{\prime} \mathscr{H}\left(t-t^{\prime}\right) \mathscr{R}_{S^{\prime} P^{\prime}}\left(z, t^{\prime}\right)
\end{aligned}
$$

When there is no dispersion, no coupling between polarizations, and the memory time is very short the equations become

$$
\begin{aligned}
& \frac{d}{d z} F_{S}(z)=-\pi \kappa_{S} \int_{-\infty}^{\infty} d t\left|\mathscr{R}_{S P}(z, t)\right|^{2} . \\
& \frac{d}{d z} F_{P}(z)=+\pi \kappa_{P} \int_{-\infty}^{\infty} d t\left|R_{P S}(z, t)\right|^{2}
\end{aligned}
$$

We recognize from these equations (and the property $\omega_{P} \kappa_{S}=\omega_{S} \kappa_{P}$ ) the condition for photon number conservation,

$$
\frac{F_{P}(z)}{\omega_{P}}+\frac{F_{S}(z)}{\omega_{S}}=\text { constant }
$$

In particular, when there is no initial Stokes seed pulse at $z=0$, then the final Stokes fluence at $z$ $\rightarrow \infty$ will be 


$$
F_{S}(\infty)=\frac{\omega_{S}}{\omega_{P}} F_{P}(0)
$$

This is the result for a pump pulse that is much longer than the memory time, and which becomes entirely converted into Stokes radiation.

\section{\$7.2 Photon Fluences; Dirac Notation}

It is useful, especially when dealing with nondispersive propagation, to introduce a form of Dirac notation with which to describe Raman processes. The notation for time integrals is:

$$
\begin{aligned}
& \langle A \mid B\rangle=\int_{-\infty}^{\infty} d t A(t)^{*} B(t) \\
& \langle A B \mid C D\rangle=\int_{-\infty}^{\infty} d t A(t)^{*} B(t) C(t)^{*} D(t)
\end{aligned}
$$

Introduce normalized photon-number amplitudes

$$
|\lambda\rangle=\sqrt{\frac{c}{8 \pi \hbar \omega_{\lambda}}} \mathscr{E}_{\lambda}(z, t) .
$$

The photon fluences and memory time are

$$
\begin{aligned}
& N_{P}(z)=\frac{c}{8 \pi \hbar \omega_{P}} F_{P}(z)=\langle P \mid P\rangle, \quad N_{S}(z)=\frac{c}{8 \pi \hbar \omega_{S}} F_{S}(z)=\langle S \mid S\rangle \\
& \tau_{R}=\langle\mathscr{G}\rangle
\end{aligned}
$$

The propagation equations for photon fluences are

$$
\begin{aligned}
\frac{d}{d z}\langle P \mid P\rangle & =-2 \mathscr{R e}\langle P \mid \mathscr{K} P\rangle \\
& -\mathcal{N}\left[\rho_{11}-\rho_{22}\right] \frac{8 \pi^{2}}{c^{2}} \omega_{P} \omega_{S} \mathscr{R e} \sum_{P^{\prime} S^{\prime} S} \alpha_{12}(P, S) \alpha_{12}\left(P^{\prime}, S^{\prime}\right)^{*}\left\langle S P \mid \mathscr{G} P^{\prime} S^{\prime}\right\rangle \\
\frac{d}{d z}\langle S \mid S\rangle & =-2 \mathscr{R e}\langle S \mid \mathscr{K} S\rangle \\
& +\mathcal{N}\left[\rho_{11}-\rho_{22}\right] \frac{8 \pi^{2}}{c^{2}} \omega_{P} \omega_{S} \mathscr{R e} \sum_{P^{\prime} S^{\prime} S} \alpha_{12}(P, S)^{*} \alpha_{12}\left(P^{\prime}, S^{\prime}\right)\left\langle P S \mid \mathscr{G} S^{\prime} P^{\prime}\right\rangle
\end{aligned}
$$


With neglect of mode coupling, and the definition $w=\rho_{22}-\rho_{11}$, these become

$$
\begin{aligned}
& \frac{d}{d z}\langle P \mid P\rangle=-2 \mathscr{R e}\langle P \mid \mathscr{K} P\rangle+\varpi \mathcal{N} \frac{8 \pi^{2}}{c^{2}} \omega_{P} \omega_{S}\left|\alpha_{12}(P, S)\right|^{2} \operatorname{Re}\langle S P \mid \mathscr{G} P S\rangle \\
& \frac{d}{d z}\langle S \mid S\rangle=-2 \operatorname{Re}\langle S \mid \mathscr{K} S\rangle-\varpi \mathcal{N} \frac{8 \pi^{2}}{c^{2}} \omega_{P} \omega_{S}\left|\alpha_{12}(P, S)\right|^{2} \mathscr{R e}\langle P S \mid \mathscr{G} S P\rangle
\end{aligned}
$$

Define a steady-state photon-fluence gain coefficient as the steady state gain for an equivalent square pulse

$$
\begin{aligned}
\bar{g}_{s s}=\mathcal{N}\left[\rho_{11}-\rho_{22}\right] \frac{8 \pi^{2}}{c^{2}} \omega_{P} \omega_{S}\left|\alpha_{12}(P, S)\right|^{2} \frac{1}{T_{P}} \frac{c}{8 \pi \hbar \omega_{P}} \int_{0}^{T_{P}} d t\left|\mathcal{E}_{P}(z, t)\right|^{2} \\
\quad=\mathcal{N}\left[\rho_{11}-\rho_{22}\right] \frac{8 \pi^{2}}{c^{2}} \omega_{P} \omega_{S}\left|\alpha_{12}(P, S)\right|^{2} \frac{1}{T_{P}}\langle P \mid P\rangle
\end{aligned}
$$

Then we can write

$$
\begin{aligned}
& \frac{d}{d z}\langle P \mid P\rangle=-2 \mathscr{R e}\langle P \mid \mathscr{K} P\rangle-\frac{g_{s s} T_{P}}{\langle P \mid P\rangle}\langle S P \mid \mathscr{G} P\rangle \\
& \frac{d}{d z}\langle P \mid S\rangle=-\langle P \mid \mathscr{K} S\rangle-\langle S \mid \mathscr{K} P\rangle^{*}+\frac{1}{2} \frac{g_{s s} T_{P}}{\langle P \mid P\rangle}[\langle P P \mid \mathscr{G} S P\rangle-\langle S S \mid \mathscr{G} S P\rangle] \\
& \frac{d}{d z}\langle S \mid S\rangle=-2 \mathscr{R} e\langle S \mid \mathscr{K} S\rangle+\frac{g_{s S} T_{P}}{\langle P \mid P\rangle}\langle P S \mid \mathscr{G} S P\rangle
\end{aligned}
$$

In steady state (short memory time) the Stokes photon fluence equation become

$$
\frac{d}{d z}\langle S \mid S\rangle=-2 \mathscr{R e}\langle S \mid \mathscr{C} S\rangle+g_{s s} T_{P} \frac{\langle P S \mid S P\rangle}{\langle P \mid P\rangle\langle S \mid S\rangle}\langle S \mid S\rangle
$$

The growth of the Stokes wave here depends on the pump-Stokes intensity correlation. For long memory time this becomes

$$
\frac{d}{d z}\langle S \mid S\rangle=-2 \mathscr{R e}\langle S \mid \mathscr{K} S\rangle+\bar{g}_{s s} T_{P}\langle\mathscr{G}\rangle \frac{1\left\langle\left. P|S|\right|^{2}\right.}{\langle P \mid P\rangle\langle S \mid S\rangle}\langle S \mid S\rangle
$$

The growth of the Stokes wave here depends on the pump-Stokes amplitude correlation. 


\section{$\S 8$ Conclusion}

We have shown that it is possible to incorporate into the traditional time-dependent coupled Maxwell-Bloch equations for Raman propagation a correction for finite bandwidth of the pump pulse.

The correction introduces an auxiliary field in place of the customary envelope. This field incorporates the same modulation as does dispersion.

The effect of this correction will depend on the nature of the pump modulation. When the modulation is slow, then the correction amounts to introducing a polarizability that follows the change in pump frequency; during those times when the instantaneous pump frequency is closer to resonance than the assumed carrier, the polarizability will be larger than the mean value.

When tine modulation is rapid, the correction has little effect, the field variation is too rapid for the atom to respond.

\section{$\S 8.2$ Acknowledgements}

This work was performed under the auspices of the United States Department of Energy $1, y$ the Lawrence Livermore National Laboratory under contract no. W-7405-Eng-48. We have benefitted from discussions with Dr. J. Paisner, Dr. T. Karr, and Dr. R. Sacks. 


\section{References}

[1] nonlinear susceptibilities

N. Bloembergen and Y. R. Shen, "Quantum-theoretical comparixon of nonlinear susceptibilities in parametric media, lasers, and Raman lasers" Phys. Rev. 133, A37-49 (1964)

D. C. Hanna, M. A. Yuratich and D. Cotter, Nonlinear Optics of Free Atoms and Molecules (Springer, Berlin, 1979)

B. J. Orr and J. F. Ward, "Perturbation theory of the non-linear optical polarization of an isolated system" Mol. Phys. 20, 513-526 (1971)

M. A. Yuratich and D. C. Hanna, "Nonlinear atomic susceptibilities" J. Phys. B. 9, 729-750 (1976)

[2] coupled maxwell bloch equations

D. Polder, M. F. H. Schuurmans and Q. H. F. Vrehen, "Superfluorescence: Quantummechanical derivation of Maxwell-Bloch description with fluctuating field source" Phys. Rev. A19, 1192-1203 (1979)

[3] SRS

N. Bloembergen "The stimulated Raman effect" Am. J. Phys 35, 989-1023 (1967)

W. Kaiser and M. Maier, "Stimulated Rayleigh, Brillouin and Raman spectroscopy" in Laser Handbook v. 2 , 1077-1050 (1972)

G. W. Chantry, "Polarizibility theory of the Raman effect" in The Raman Effect ed. A. Anderson, v. 1, ch 2, 49-94 (Dekker, 1971)

R. W. Hellwarth, "Theory of stimulated Raman scattering" Phys. Rev. 130, 1850-52 (1963)

P. Lallemand, "The Stimulated Raman effect" in The Raman Effect ed. A. Anderson, v. 1, ch. 5, 287-342 (Dekker, 1971)

Y. R. Shen and N. Bloembergen, "Theory of stimulated Brilloun and Raman scattering" Phys. Rev. 137, A1787-1805 (1965) 

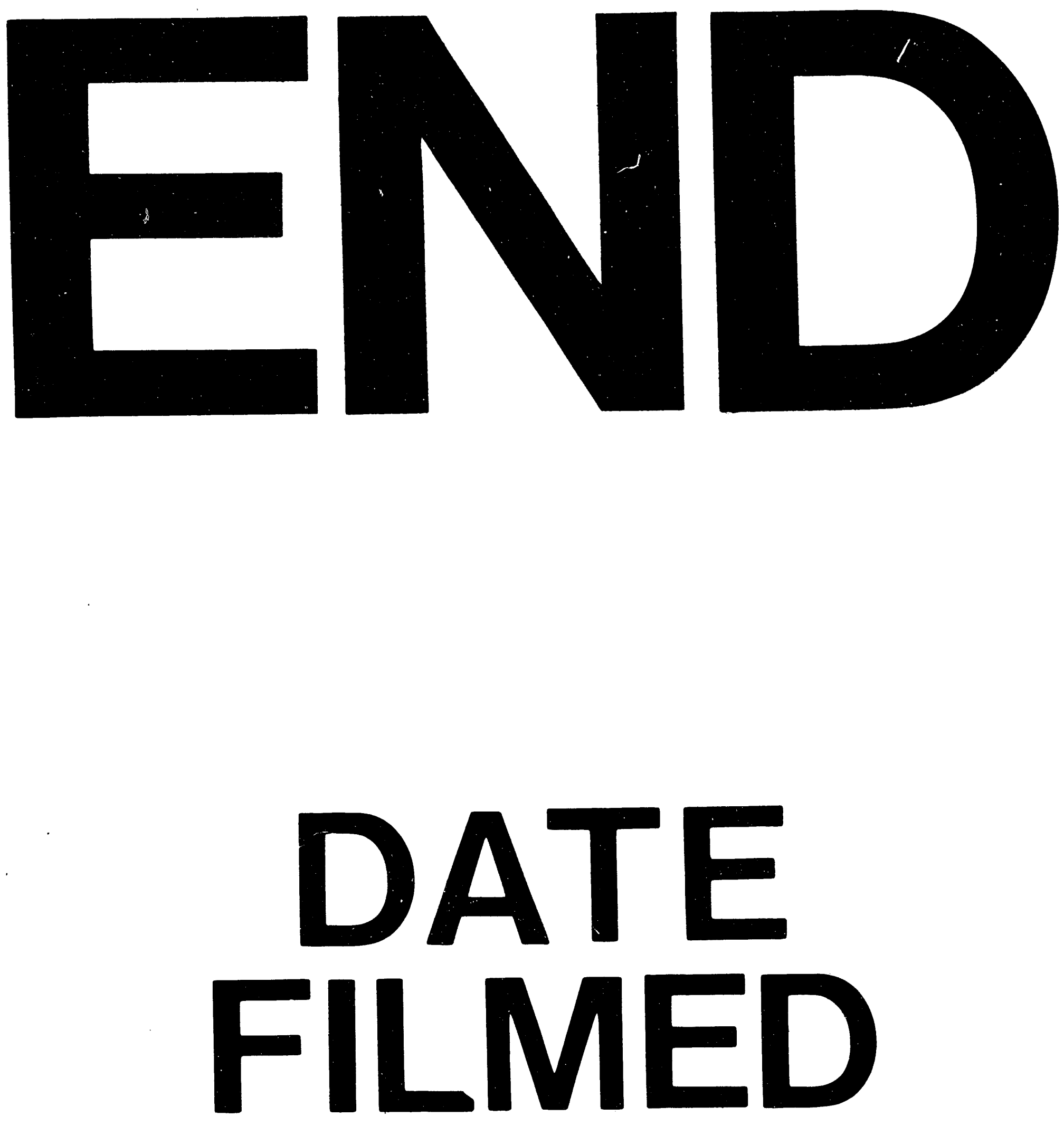

1

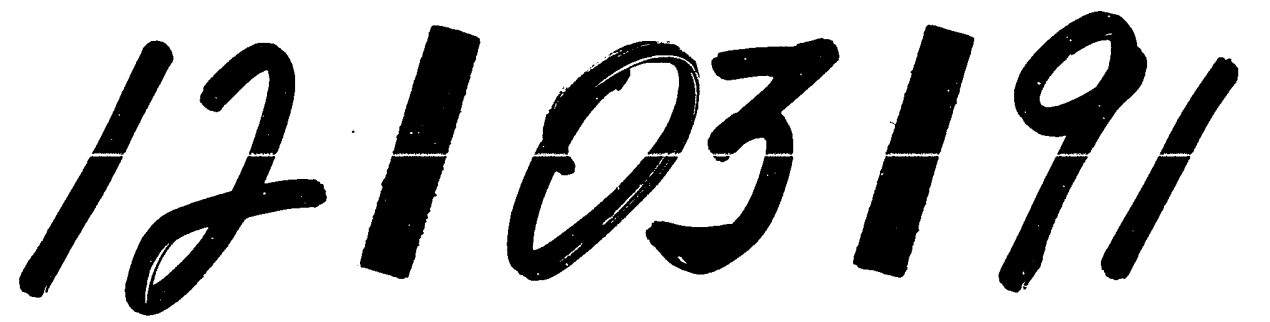


